First Version: April 2001. This Version June 2002.

\title{
Equilibrium asset pricing with time-varying pessimism
}

\begin{abstract}
We present a flexible analytical framework that incorporates the equilibrium impact of a (possibly state dependent) sentiment for pessimism in continuous time intertemporal asset pricing. State dependent pessimism comes from a state dependent confidence in the reference belief on equity returns dynamics and implies conservative optimal policies precisely in states where such confidence is low. In the setting of an homogeneous exchange economy we characterize analytically the impact of pessimism on key equilibrium quantities, including the implied equity dynamics, the equilibrium interest rate and the corresponding equity premia and worst case equity premia. Due to the complexity of the implied optimization problems we adopt a perturbative approach to compute finite order approximations for these quantities. We find that pessimism induces directly lower equilibrium interest rates and higher equity premia, while it impacts only indirectly - through the interplay of risk and model uncertainty aversion - the expected returns on equity, their variances and covariances with the opportunity set process and the implied worst case equity premia. Thus, to first order these latter variables are fully determined by the standard risk aversion parameter. We compute some higher order asymptotics attempting to quantify the direct and indirect impacts of pessimism in equilibrium for several examples of a pessimistic exchange economy with intermediate consumption and stochastic opportunity set. For all these examples we find that the indirect impact of pessimism on equity dynamics and worst case equity premia is negligible, while the influence on equity premia and interest rates is quantitatively significant already for moderate amounts of pessimism. This confirms the results implied by a pure first order analysis. Therefore, in the setting of a representative agent exchange economy a pessimistic concern for model uncertainty can help explaining part of the equity premium puzzle while maintaining low interest rates and leaving virtually unaffected some target parameters in the equilibrium equity dynamics.
\end{abstract}

\author{
Alessandro Sbuelz \\ Department of Finance, Tilburg University, P.O. Box 90153, 5000 LE Tilburg, The Netherlands, e-mail: \\ A.Sbuelz@kub.nl \\ Fabio Trojani \\ Institute of Finance, University of Southern Switzerland, Via Buffi 13, CH-6900 Lugano, \\ e-mail: Fabio.Trojani@lu.unisi.ch
}




\title{
Equilibrium asset pricing with time-varying pessimism
}

\begin{abstract}
We present a flexible analytical framework that incorporates the equilibrium impact of a (possibly state dependent) sentiment for pessimism in continuous time intertemporal asset pricing. State dependent pessimism comes from a state dependent confidence in the reference belief on equity returns dynamics and implies conservative optimal policies precisely in states where such confidence is low. In the setting of an homogeneous exchange economy we characterize the impact of pessimism on key equilibrium quantities, including the implied equity dynamics, the equilibrium interest rate and the corresponding equity premia and worst case equity premia. Due to the complexity of the implied optimization problems we adopt a perturbative approach to compute finite order approximations for these quantities. We find that pessimism induces directly lower equilibrium interest rates and higher equity premia, while it impacts only indirectly - through the interplay of risk and model uncertainty aversion - the expected returns on equity, their variances and covariances with the opportunity set process and the implied worst case equity premia. Thus, to first order these latter variables are fully determined by the standard risk aversion parameter. We compute some higher order asymptotics attempting to quantify the direct and indirect impacts of pessimism in equilibrium for several examples of a pessimistic exchange economy with intermediate consumption and stochastic opportunity set. For all these examples we find that the indirect impact of pessimism on equity dynamics and worst case equity premia is negligible, while the influence on equity premia and interest rates can be significant already for moderate amounts of pessimism.. This confirms the results implied by a pure first order analysis. Therefore, in the setting of a representative agent exchange economy a pessimistic concern for model uncertainty can help explaining part of the equity premium puzzle, while maintaining low interest rates and leaving virtually unaffected some target parameters in the equilibrium equity dynamics.
\end{abstract}

JEL Classification: G11, G12

Keywords: Asset Pricing, General Equilibrium, Model Misspecification, Perturbation Theory, Knightian Uncertainty. 


\section{Contents}

1 Introduction $\quad 1$

2 The economy $\quad 3$

2.1 Assets, cash flow, and state variables . . . . . . . . . . . . . . . . . . 4

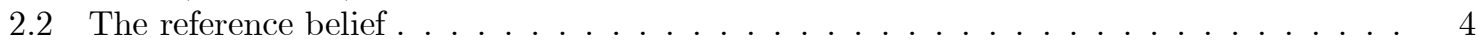

2.3 Scenarios . . . . . . . . . . . . . . . . . . . . . . 4

2.4 Risk aversion and concern for model uncertainty . . . . . . . . . . . . . . . . 5

2.5 Pessimistic maxmin utilities and worst case scenarios . . . . . . . . . . . . . . . 6

3 Partial equilibrium perturbative solutions $\quad 6$

3.1 The perturbative approach . . . . . . . . . . . . . . . . . . . 7

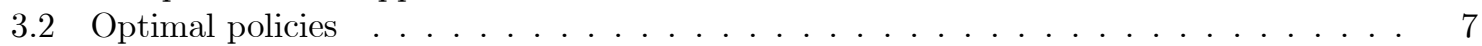

4 Pessimistic exchange equilibrium $\quad 9$

4.1 Representative agent's equilibrium value function . . . . . . . . . . . . . . . . . 10

4.2 Equilibrium cumulative returns on equity . . . . . . . . . . . . . . . . . . . . . . . . . . . . . . . . .

4.3 Equilibrium equity premia and interest rates . . . . . . . . . . . . . . . . . . . 15

4.4 Higher order properties of pessimistic value functions . . . . . . . . . . . . . . . 16

5 Some explicit settings of exogenous pessimism $\quad 20$

$5.1 O\left(\gamma^{3}\right)$-equilibrium asymptotics . . . . . . . . . . . . . . . . . . . . . . . . . . . . . . . . . . . .

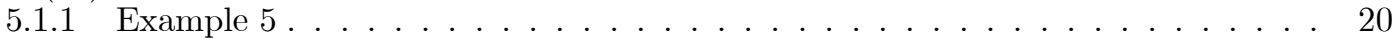

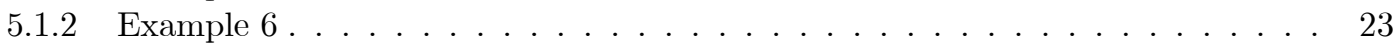

5.1 .3 Example $15 \ldots \ldots \ldots \ldots \ldots \ldots$

$5.2 O^{2}(\gamma, \sqrt{\varphi})$-equilibrium asymptotics . . . . . . . . . . . . . . . . . . 26

5.2.1 Example 5 with state dependent pessimism . . . . . . . . . . . . . . . 26

5.2.2 Example 6 with a further parameterization of pessimism . . . . . . . . . 28

6 Conclusions $r$

$\begin{array}{llr}7 & \text { Appendix } & 30\end{array}$ 


\section{List of Figures}

1 Ornstein Uhlenbeck (OU) dynamics: Equity premia $\left(O\left(\gamma^{3}\right)\right.$-equilibrium

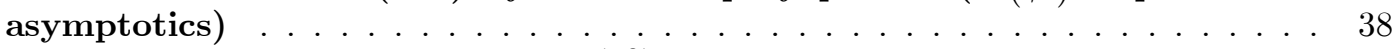

2 OU dynamics: Equity returns $\left(O\left(\gamma^{3}\right)\right.$-equilibrium asymptotics) and func-

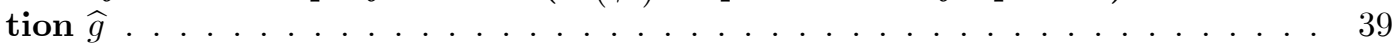

3 Cox Ingersoll Ross (CIR) dynamics: Equity premia $\left(O\left(\gamma^{3}\right)\right.$-equilibrium

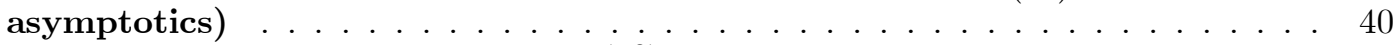

4 CIR dynamics: Equity returns $\left(O\left(\gamma^{3}\right)\right.$-equilibrium asymptotics) and func-

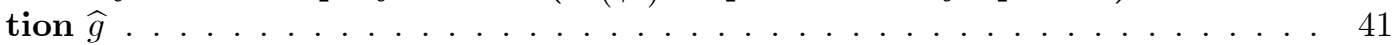

5 Geometric Ornstein Uhlenbeck (GOU) dynamics: Equity premia $\left(O\left(\gamma^{3}\right)\right.$-equilibrium

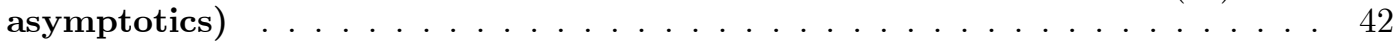

6 GOU dynamics: Equity returns $\left(O\left(\gamma^{3}\right)\right.$-equilibrium asymptotics) and func-

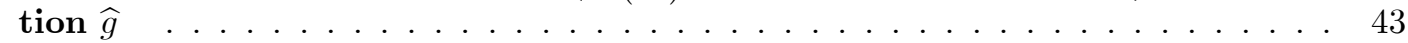

7 OU dynamics: Equity premia $\left(O^{2}(\gamma, \sqrt{\varphi})\right.$-equilibrium asymptotics $) \quad \ldots \quad 44$

8 CIR dynamics: Equity premia $\left(O^{2}(\gamma, \sqrt{\varphi})\right.$-equilibrium asymptotics $) \ldots \ldots$ 


\section{Introduction}

We study analytically the equilibrium asset pricing impact of a (possibly time varying) pessimistic concern for model uncertainty in a representative agent exchange economy with intermediate consumption and stochastic opportunity set. Pessimistic investors are assumed to act as if under the worst case expected return on equity that arises from a time-varying agent's distrust about some reference expected returns. This implies conservative policies especially in states where such distrust is high. We compute the general equilibria implied by pessimistic economies with state-dependent opportunity set and intertemporal consumption-portfolio choice and find that pessimism reduces equilibrium interest rates and enhances equity premia in a direct and quantitatively important way, under both plausible assumptions on the model parameters and realistic amounts of pessimism. By contrast, the equilibrium equity dynamics and worst case equity premia are only marginally (and indirectly) affected by pessimism.

The asset pricing impact of pessimism in our models comes from a specific economic mechanism (Knight's (1921) uncertainty aversion) and has a specific size (first order risk aversion - FORA - effect). This incarnates in a high equity premium even when equity risk is low, because Knightian (1921) aversion to model misspecification and FORA imply pessimistic equity premia that are proportional to equity volatility ${ }^{1}$. This is economically and observationally specific to our approach. Classic expected utility, habit formation preferences, catching-up-with-the-Joneses preferences, Epstein-Zin preferences, and alternative pessimistic preferences are not carachterized by Knightian aversion to model misspecification and produce equity premia that are proportional to equity variance. The link between a time varying degree of Knightian pessimism and the state of the economy is meant to add flexibility. For example, it turns useful when employing our approach to link time varying pessimism with some business-cycle-based forecast of the excess returns on equity.

The motivation for considering agent's confidence in their beliefs comes from simple introspection and Ellsberg's (1961) paradox. The latter provides evidence that misspecification risk is an actual concern because investors show aversion to it: They seem to dislike gambles with unknown probability law. The modelling hypothesis of a time-varying structure for pessimism is supported by the empirical evidence that investors tend to be more uncertain about the future growth of the economy during recessions. For example, Veronesi (1999) reports that economists' forecasts on the future real output growth (taken from the US Livingston survey) are more dispersed - that is, they have greater crosssectional standard deviation - when the economy is contracting. In our setting, such a time-varying pessimism is the further trasmission mechanism between economic fundamentals and time-varying excess expected returns.

The predictability literature based on business-cycle indicators further motivates our hypothesis of time-varying pessimism. A large empirical literature documents that expected excess returns and equity return volatilities are higher in recessions. For instance, Fama and French (1989) highlight that expected excess returns vary over business cycles as only a higher risk premium gets people to hold equity at the bottom of a recession. In further related research, Ferson and Harvey (1991) and Harvey (1991) provide empirical evidence on countercyclical variations in the market price of risk and in expected excess returns, as they are negatively related to the price-dividend ratio and are higher during business-cycle troughs than during peaks. Cochrane (2000) surveys the literature to conclude that the price-dividend ratio and any other variable correlated with the business cycle help in explaining expected excess returns. Such reported evidence backs the intuition that investors are more pessimistic during recessions, thus showing countercyclical effective risk aversion. Our setting

\footnotetext{
${ }^{1}$ Dow and Werlang (1992) provide a general discussion on FORA effects.
} 
of time varying pessimism can generate such a countercyclical risk aversion behavior by positing that investors fear equity precisely when they are more uncertain about the underlying economic fundamentals.

Countercyclical risk aversion arises also from settings without pessimism. Recently, Campbell and Cochrane (1999) have proposed a model with countercyclical state-dependent risk aversion and habit formation to explain many of the empirical observed features of asset prices. In a related paper Chan and Kogan (2001) show that in a general equilibrium economy with heterogeneous catching-upwith-the-Joneses preferences, the endogenous dynamics of cross-sectional wealth can generate countercyclical time variation in the Sharpe ratio of equity returns. By contrast, in our paper agents are endowed with a standard CRRA utility function. However, they behave in a pessimistic way by computing worst case expected utilities of future consumption, where the worst case is a function of a state dependent degree of confidence in some given reference belief. This generates behaviorally and observationally different optimal policies through FORA effects. More specifically, in our setting investors formulate a reference belief about equity returns dynamics and consider, in making their portfolio-consumption decisions, scenarios around that reference belief. Scenarios represent model uncertainty for our investor and are allowed to depend on the state of the economy, so that agent's aversion to model uncertainty becomes state dependent. This is a simple framework that combines a state dependent reference belief about the opportunity set, state dependent scenarios around it, and a state dependent aversion to that form of model uncertainty. Investors maximize their expected utility with respect to the worst case scenario out of the set of relevant scenarios. Since scenarios can be state dependent, this framework is consistent with a time varying confidence in the reference belief. Therefore, in our model maxmin expected utility maximization implies equilibrium equity premia that mirror a state dependent confidence in the reference belief.

A high equity premium in the long run is not necessarily linked with unrealistically high risk aversions in our setting. Indeed, in our framework while classical risk aversion determines expected equity returns, aversion to model uncertainty pushes down the instantaneous riskless rate. The sustained demand for instantaneous discount bonds is driven by pessimism about equity. At the same time, willingness of substituting consumption intertemporally is kept constant. Thus, we can separate the impact of pessimism from the one induced by the elasticity of intertemporal substitution. After Duffie and Epstein (1992), scholars have been using continuous-time settings with stochastic differential utility (SDU) to increase risk aversion while keeping fixed the elasticity of intertemporal substitution. Our framework is economically and observationally different from SDU as we focus on model uncertainty and because in our model pessimism has a FORA effect on asset substitution. Models of intertemporal robust decision making (for example, Anderson, Hansen, and Sargent (2000), Maenhout (1999)) are also economically and observationally different from our framework. They differ from our setting by introducing a form of pessimism that yields optimal policies observationally equivalent to those implied by SDU, thus generating second order risk aversion. Similarly, the definition of pessimism and doubt in the discrete time asset pricing model of Abel (1997) and the multy-asset setting with model uncertainty in Uppal and Wang (2000) generate pessimistic asset price relationships that mimic second order risk aversion.

Our setting captures model ambiguity in the sense of Chen and Epstein (2000). Chen and Epstein (2000) consider an intertemporal extension of the multiple-priors atemporal ambiguity model under maxmin behavior in Gilboa and Schmeidler (1989). In particular, this extension is the only one that enjoys dynamic consistency in the standard sense of Johnsen and Donaldson (1985). This makes sure that investors will not take investment decisions against their future selves. The specific form of multiple priors we use is characterized by a continuity of indistinguishable scenarios around the reference belief. We interpret agent's confidence in the reference belief as the inverse of the maximal discrepancy, that is, the discrepancy between the worst case scenario and the reference belief. Agent's maxmin preferences imply pessimistic portfolio choices with respect to the whole neighborhood of relevant scenarios. 
Indistinguishability of scenarios and presence of pessimism make our framework different also from intertemporal Bayesian settings ${ }^{2}$ of equilibrium asset pricing like Veronesi (1999), Veronesi (2000), and Veldkamp (2001). Such settings are based on a single prior - for example, conditional expected dividends have a given finite state space with given transition probabilities in Veronesi (1999) and Veronesi (2000) - and capture essentially parameter uncertainty, that is estimation risk. By contrast, in our setting investors acknowledge their inability to learn much through time (in a Bayesian sense) about the sharpness of their reference beliefs. The best they can do is to approximately calibrate such beliefs using the available data information and to consider a set of indistinguishable scenarios around the reference belief, when determinig optimal consumption/investment plans ${ }^{3}$. Thus, they adopt a pessimistic maxmin utility approach which generates FORA effects that are absent in standard Bayesian models based on a single prior.

The maxmin utility behavior in our model induces optimality conditions and optimal policies that are even less analytically tractable than their standard expected utility-based counterparts: Exact optimal policies are not computable in closed form and exact analytical characterizations of the implied equilibria are impossible. We therefore adopt a perturbative approach that permits us to analyze analytically the general equilibria implied by our setting of pessimistic decision making. Specifically, we perturb the general equilibria of a benchmark economy with homogeneous (pessimistic or not pessimistic) log utility agents are and we produce finite order approximations for the desired equilibrium quantities. The perturbation parameters are the relative risk aversion or the degree of pessimism parameters in our economies. This extends the perturbative methodology in Kogan and Uppal (2000) for standard (non pessimistic) Merton's (1969, 1971)-type models, in order to account for model vagueness in intertemporal portfolio-consumption choice.

Our asymptotics show that pessimism induces directly lower equilibrium interest rates and higher equity premia, while it impacts only indirectly - through the interplay of risk and model uncertainty aversion - the expected returns on equity, their variances and covariances with the opportunity set process and the implied worst case equity premia. Specifically, to first order these latter variables are fully determined by the standard risk aversion parameter. Moreover, the higher order impact of pessimism on the equity dynamics and worst case equity premia is negligible while the direct effect on equity premia and interest rates is quantitatively significant. Indeed, for amounts of pessimism that correspond to high probabilities of a model detection error (cf. Anderson, Hansen and Sargent (2000)) the implied FORA effect on equity premia already has an important size. Therefore, in the setting of a representative agent exchange economy with CRRA utility function a pessimistic concern for model uncertainty can help explaining part of the equity premium puzzle, while maintaining low equilibrium interest rates and leaving virtually unaffected some target parameters in the equilibrium equity dynamics.

The paper is organized as follows. Section 2 defines the key ingredients of our pessimistic economies. Section 3 provides the first order asymptotics for the implied partial equilibrium optimal policies. In Section 4 we compute the corresponding general equilibrium expansions for an abstract exchange economy with state dependent opportunity set and time varying pessimism. Section 5 presents some explicit settings of time varying pessimism and Section 6 concludes.

\section{The economy}

In this section we introduce the basics of our pessimistic economies, starting from the reference model dynamics that represents agent's approximate description of asset prices, going further to define the

\footnotetext{
${ }^{2}$ For a review of continuous-time Bayesian models of optimal portfolio choice, see Veronesi (2000).

${ }^{3}$ See p. 4 in subsection 1.2 of Chen and Epstein (2000) for more details on this "no learning" assumption.
} 
class of relevant scenarios around the reference belief, and concluding with the maxmin expected utility optimization problem that generates pessimistic optimal policies in our model.

\subsection{Assets, cash flow, and state variables}

There are two long-lived assets, a discount bond - the riskfree asset with instantaneous rate $r$ - and equity - the risky asset with ex-dividend price $P$ - which is a claim on a dividend process $e$. Riskfree rate, expected dividend growth and dividend growth volatility constitute agent's opportunity set. The opportunity set is governed by the opportunity set process $X$ which drives agent's reference belief on the economy. Pessimism is described by a (possibly vector valued) state variable that synthesizes the relevant state of the economy. This variable's dynamics follows either a meta-process (investors have no ambiguity on it) or the opportunity set process itself, and drives agent's confidence on the reference belief, that is, the maximal discrepancy between the reference belief and scenarios.

\subsection{The reference belief}

The reference belief on the dynamics of the opportunity set process, dividend growth, and cumulative returns to equity, respectively, is

$$
\begin{aligned}
d X & =\zeta(X) d t+\xi(X) d Z^{X} \\
\frac{d e}{e} & =\alpha_{e}(X) d t+\sigma_{e}(X)\left[\rho_{e}(X) d Z^{X}+\sqrt{1-\rho_{e}^{2}(X)} d Z^{e}\right] \\
\frac{d P+e d t}{P} & =\alpha_{P} d t+\sigma_{P}\left[\rho d Z^{X}+\sqrt{1-\rho^{2}} d Z^{e}\right]
\end{aligned}
$$

where $Z=\left(Z^{X}, Z^{e}\right)^{\prime}$ is a bivariate standard Brownian Motion with mutually independent components. Cumulative returns on equity have conditional expectation $\alpha_{P}$, conditional volatility $\sigma_{P}$, and conditional correlation coefficient $\rho$ to be determined in equilibrium. The instantaneous riskless rate $r$ is determined in equilibrium as well. The couple $\left(\alpha_{P}, \sigma_{P}\right)$ represents the risk / return profile offered by equity to investors. The opportunity set process $X$ represents the exogenous primitives in our economy. This is why we choose to represent the other processes in the economy as driven by $d Z^{X}$, the standardized news on the opportunity set changes $d X$, and by idiosyncratic news. Investors place a fraction $w$ of their individual wealth $W$ to equity and a fraction $c$ to current consumption. Thus, the dynamics of the vector $Y=(X, W)^{\prime}$ is

$$
d Y=\mu d t+\Lambda d Z
$$

where

$$
\mu=\left(\begin{array}{c}
\zeta \\
w W\left(\alpha_{P}-r\right)+W(r-c)
\end{array}\right), \quad \Lambda=\left[\begin{array}{cc}
\xi & 0 \\
\rho w W \sigma_{P} & \sqrt{1-\rho^{2}} w W \sigma_{P}
\end{array}\right]
$$

\subsection{Scenarios}

Scenarios are generated by local contaminations of the reference belief. Let $\nu$ be the Radon-Nikodym derivative of a contaminated probability law with respect to the reference probability law. $\eta$ is $\nu$ 's best forecast at time $t$ so that

$$
\frac{d \eta}{\eta}=h^{\prime} d Z, \quad h=\left(h^{X}, h^{P}\right)^{\prime}, \quad E(\eta)=1 .
$$

$E\left(E_{t}\right)$ is the unconditional (conditional) expectation operator under the reference probability law induced by the reference belief. $\eta$ is the scaling factor that generates scenarios around the reference 
belief. Once $Y$ is scaled by $\eta$, its annualized reference drift is added with a vector of unknown time-varying contaminations

$$
\frac{1}{d t} E_{t}\left(\frac{1}{\eta} d(\eta Y)\right)=\frac{1}{d t} E_{t}^{h}(d Y)=\mu+\Lambda h .
$$

$E^{h}\left(E_{t}^{h}\right)$ is the unconditional (conditional) expectation operator under the probability law induced by the contaminated scenario referring to a specific $\nu$. No learning whatsoever can be done on the contaminating vector $h$. $h$ is premultiplied by the volatilities matrix $\Lambda$ because it comes about as a Girsanov-like change of drift. This is a technical detail of our continuous-time setting which by no means determines FORA: FORA derives from the maxmin utility behavior of investors with constrained maximal distrust in their reference beliefs; cf. Section 2.4 below.

\subsection{Risk aversion and concern for model uncertainty}

Investors have time preference rate $\delta$ and get the following Constant Relative Risk Aversion (CRRA) utility $u(\cdot)$

$$
u(c W)=\frac{(c W)^{\gamma}-1}{\gamma} ; \gamma<1
$$

out of current consumption $\mathrm{cW}$. As usual for $\gamma \rightarrow 0$ the log utility case arises. The coefficient of relative risk aversion is constant and equal to $1-\gamma$.

Agent's attitudes towards model misspecification are described by a maximal bound $\varphi f^{2}(X)$ on the size of a relevant contaminating vector $h$,

$$
\frac{1}{2} h^{\prime} h \leq \varphi f^{2}(X)
$$

where $\varphi$ is a non-negative constant and $f$ a function that relates the current state $X$ of the economy to the inverse of investors confidence in the given reference belief. Setting $f(X)$ equal to some simple function of $X$ can be helpful in producing a parsimonious model specification in primitive parameters. Candidates for the process $f(X)$ can be for example indicators of expected dividend growth and dividend growth volatility. Furthermore, remark that further exogenous state variables in excess of $X$ can be included in the definition of the bound (2) without changing significantly the derivations that follow ${ }^{4}$. We do not elaborate further on this point here for simplicity of exposition.

For $\varphi$ decreasing to 0 , investors tend to have full confidence in their reference belief on equity dynamics. For $\varphi$ greater than 0 , investors consider a continuity of scenarios around the reference belief. These scenarios form a whole neighborhood of contaminated models centered in the reference model. The maximal bound $\varphi f^{2}(X)$ defines the radius of such a neighborhood. Such maximal bound is state-dependent via the variable $X$ that defines, through the function $f$, the impact of the state of the economy on agent's confidence in her reference belief. For instance, the free structure of $f$ can capture possible asymmetries of the impact of the state of the economy on agent's faith in her reference model. Equivalently, investors relevant scenarios are described by a maximal bound $\varphi f^{2}(X)$ on the rate at which relative entropy of a relevant model misspecification is allowed to increase over time,

$$
\frac{1}{d t} E_{t}\left(\frac{1}{\eta} d(\eta \ln \eta)\right)=\frac{1}{d t} E_{t}^{h}(d \ln \eta)=\frac{1}{2} h^{\prime} h \leq \varphi f^{2}(X) .
$$

Thus, the maximal bound $\varphi f^{2}(X)$ represents the inverse of agent's confidence in the reference belief as it measures the discrepancy between the worst case scenario and the reference belief. In the next section we define how agent's maxmin preferences imply portfolio choices that are pessimistic with respect to the whole neighborhood of relevant scenarios.

\footnotetext{
${ }^{4}$ Such an extended model setting can be useful to model a pessimism process that is not fully correlated with the starting reference model primitives.
} 


\subsection{Pessimistic maxmin utilities and worst case scenarios}

For a utility function $u$ of the form (1) the value function of our pessimistic investor is given by

$$
J(Y)=\left\{\begin{array}{c}
\max _{c, w} \min _{h} E_{0}^{h}\left[\int_{0}^{\infty} e^{-\delta t} u(c W) d t\right] \\
\frac{1}{2} h^{\prime} h \leq \varphi f^{2}(X) \quad, \quad d Y=\mu d t+\Lambda d Z
\end{array} .\right.
$$

This yields the Hamilton-Jacobi-Bellman (HJB) equation

$$
\left\{\begin{array}{c}
0=\max _{c, w} \min _{h}\left(u(c W)-\delta J+\frac{1}{d t} E_{t}^{h}(d J)\right) \\
\frac{1}{2} h^{\prime} h \leq \varphi f^{2}(X) \quad, \quad d Y=\mu d t+\Lambda d Z
\end{array} .\right.
$$

Under a given scenario alternative $h$ to the reference belief, the annualized expected change in the value function is

$$
\frac{1}{d t} E_{t}^{h}(d J)=\frac{1}{d t} E_{t}(d J)+h^{\prime} \Lambda^{\prime} J_{Y}
$$

where $J_{Y}$ is $J$ 's gradient with respect to $Y$. Such a structure for the HJB problem unveils the structure of the worst case scenarios. They come from the constrained minimization with respect to the contaminating vector $h$. The Lagrangean solution to it is standard ${ }^{5}$ (cf. also AHS (1998) and Trojani and Vanini (2002)).

Proposition 1 The worst case contaminating vector is

$$
h^{*}=-\frac{\sqrt{2 \varphi}|f(X)|}{\left(J_{Y}^{\prime} \Lambda \Lambda^{\prime} J_{Y}\right)^{\frac{1}{2}}} \Lambda^{\prime} J_{Y} .
$$

Notice that the worst case contaminating vector $h^{*}$ is a vector of norm equal to the maximal allowed discrepancy $\sqrt{2 \varphi}|f(X)|$. Further, $h^{*}$ downweights the worst case expected change ${ }^{6}$

$$
\frac{1}{d t} E_{t}^{h^{*}}(d J)=\frac{1}{d t} E_{t}(d J)+h^{* \prime} \Lambda^{\prime} J_{Y}=\frac{1}{d t} E_{t}(d J)-\sqrt{2 \varphi J_{Y}^{\prime} \Lambda \Lambda^{\prime} J_{Y}}|f(X)|
$$

in the value function $J$ precisely when either the volatility $\sqrt{J_{Y}^{\prime} \Lambda \Lambda^{\prime} J_{Y}}$ of $d J$ is large or when a higher model uncertainty (that is a higher maximal allowed discrepancy $\sqrt{2 \varphi}|f(X)|$ ) causes a lower confidence in the reference model.

\section{Partial equilibrium perturbative solutions}

In this section we analyze the structure of the partial equilibrium optimal policies of our pessimistic investor and discuss analytically the differences with standard (non pessimistic) portfolio policies. We obtain this using a set of perturbed asymptotics for the implied value function and optimal policies. The perturbation parameters are the risk aversion index $\gamma$ and the degree of pessimism $\sqrt{\varphi}$ in our economies. Thus, we approximate the desired partial equilibrium quantities in a neighborhood of the solution of a standard (non pessimistic) log utility agent. This section focuses on first order approximations that highlight the partial equilibrium effects of FORA on the implied pessimistic optimal policies.

\footnotetext{
${ }^{5}$ A proof is given for completeness in the appendix.

${ }^{6}$ We denote the worst case conditional expectation operator by $E_{t}^{h *}(\cdot)$.
} 


\subsection{The perturbative approach}

Homogeneity of the HJB problem leads to the following educated guess on J's functional form,

$$
J(X, W)=\frac{1}{\delta} \frac{\left(e^{g(\gamma, \sqrt{\varphi}, X)} W\right)^{\gamma}-1}{\gamma} .
$$

The function $g$ expresses how investors welfare is affected by intertemporal changes in their reference belief on the opportunity set and in their confidence about such belief. Thus, $g$ 's derivatives with respect to $X$ determine agent's intertemporal hedging policies. Finding a closed form for $g$ is typically wishful thinking so that these problems are analytically intractable. We use a perturbative way around it pioneered by Kogan and Uppal (2000) and expand the function $g$ in terms of the parameter vector ${ }^{7}$ $(\gamma, \sqrt{\varphi})^{\prime}$

$$
g(X)=g_{0}(X)+\gamma g_{1}(X)+\sqrt{\varphi} g_{2}(X)+O^{2}(\gamma, \sqrt{\varphi}) \quad,
$$

where $g_{0}=g_{0}(X)$ is determined by the value function solution of the constrained maxmin problem (3) for $\gamma, \sqrt{\varphi} \rightarrow 0$. This is the solution when investors have log utility and place full faith in their reference belief. Denoting by $J_{\log }(X, W)$ this solution it follows

$$
J_{\log }(X, W)=\frac{1}{\delta}\left(\ln (W)+g_{0}(X)\right) .
$$

Notice that explicit knowledge of $J_{\log }$ implies explicit knowledge of $g_{0}$ and that the standard logarithmic case is more tractable that the initial maxmin optimization. A remarkable result of this perturbative approach is that $g_{0}$ fully characterizes agent's optimal rules, to first order in $(\gamma, \sqrt{\varphi})$. Moreover, standard logarithmic investors do not suffer of misconfidence in their reference belief so that they are only concerned about the direct effects of the state variable $X$ on the opportunity set. This in turn implies that the part of optimal portfolio holdings motivated by an intertemporal hedging against changes in agent's pessimism is of second order in $\gamma, \sqrt{\varphi}$; cf. also the next section.

\subsection{Optimal policies}

We define the following two latent risk factors

$$
\underbrace{\kappa=\frac{\alpha_{P}-r}{\sigma_{P}}}_{\begin{array}{c}
\text { Price of Equity Risk } \\
\text { (Sharpe ratio) }
\end{array}}, \quad \underbrace{\psi=\xi \frac{\partial g_{0}}{\partial X}}_{\begin{array}{c}
\text { Agent's Approximate Sensitivity to } \\
\text { Stochastic Opportunity Set }
\end{array}}
$$

They will assist us in the interpretation of the implied perturbative optimal policies, as they are the basic first-order components of both pessimistic (maxmin) and standard (expected utility) consumption/portfolio choices. Indeed, it is well known (Merton (1971)) that an expected utility investor $(\varphi=0)$ chooses an optimal portfolio that is made of the sum of a myopic portfolio and an hedging portfolio. The myopic portfolio reflects agent's speculative attitude. Thus, it is related to equity's expected reward per unit of risk, that is, it is related to the price of risk $\kappa$. The hedging portfolio reflects agent's willingness to insure against future adverse movements in their

${ }^{7}$ Hereafter $O^{2}(\gamma, \sqrt{\varphi})$ is a symbol that we use to denote terms of second order in $(\gamma, \sqrt{\varphi})$, that is,

$$
O^{2}(\gamma, \sqrt{\varphi})=O\left(\|(\gamma, \sqrt{\varphi})\|^{2}\right)
$$

where $\|(\cdot, \cdot)\|$ is the Euclidean norm of the vector $(\cdot, \cdot)$. 
risk return profile $\left(\alpha_{P}, \sigma_{P}, \rho\right)$ due to changes in the underlying state variables. Thus, it is related to how $d X$-driven changes in $\left(\alpha_{P}, \sigma_{P}, \rho\right)$ affect agent's value function, that is it is related to agent's sensitivity $\psi$ to changes in the stochastic opportunity set. The standard myopic and hedging portfolios can be approximated by

$$
w^{M}(X)=\frac{\kappa}{\sigma_{P}(1-\gamma)} \quad, \quad w^{H}(X)=\frac{\rho \psi \gamma}{\sigma_{P}(1-\gamma)} \quad,
$$

respectively, since $w^{H}(X)$ is already a $\gamma$-first order approximation for the hedging demand of a standard investor ${ }^{8}$ with relative risk aversion $1-\gamma$. The two latent risk factors $\kappa$ and $\psi$ depend only on the opportunity set state $X$. The next proposition shows that $\kappa$ and $\psi$ determine completely the first order corrections for $w^{M}(X)$ and $w^{H}(X)$, which a pessimistic investor is going to implement ${ }^{9}$.

Proposition 2 The optimal consumption and investment policies to problem (3) are given by

$$
\begin{aligned}
c^{*}(X) & =\delta\left(1-\gamma\left(g_{0}(X)-\ln (\delta)\right)\right)+O^{2}(\gamma, \sqrt{\varphi}) \\
w^{*}(X) & =w^{M}(X ; \varphi)+w^{H}(X ; \varphi)+O^{2}(\gamma, \sqrt{\varphi})
\end{aligned}
$$

where

$$
\begin{aligned}
w^{M}(X ; \varphi) & =w^{M}(X) \frac{1}{1+\frac{1}{1-\gamma} \sqrt{\frac{2 \varphi}{\kappa^{2}+\psi^{2}+2 \rho \kappa \psi}}|f(X)|} \\
w^{H}(X ; \varphi) & =w^{H}(X) \frac{1-\frac{1}{\gamma} \sqrt{\frac{2 \varphi}{\kappa^{2}+\psi^{2}+2 \rho \kappa \psi}}|f(X)|}{1+\frac{1}{1-\gamma} \sqrt{\frac{2 \varphi}{\kappa^{2}+\psi^{2}+2 \rho \kappa \psi}}|f(X)|}
\end{aligned}
$$

From Proposition 2 we notice first that to first order pessimism does not affect optimum consumption. Indeed, $c^{*}$ is the same optimal consumption ratio that would be selected in our model by a standard expected utility agent $(\varphi=0)$. By contrast, pessimism influences both the myopic and the hedging demands for equity in a state dependent way, via the latent risk factors $\kappa, \psi$, and the pessimism index $|f(X)|$. Specifically, state dependence through $\kappa$ and $\psi$ reflects the direct impact of a concern for model misspecification in the dynamics of the opportunity set process $X$. On the other hand, $|f(X)|$ describes the way by which time variation in pessimism affects optimal portfolios.

The resulting demand for equity can be interpreted as the one that would be implemented by an investor with a state dependent effective relative risk aversion

$$
1-\left(\gamma-\sqrt{\frac{2 \varphi}{\kappa^{2}+\psi^{2}+2 \rho \kappa \psi}}|f(X)|\right)
$$

that penalizes states where model misspecification can strongly reduce the performance of a portfolio strategy. Such state dependent effective risk aversion affects both the myopic and the hedging demand for equity and cause FORA effects. Depending on the joint structure of $X$ 's and $f(X)$ 's dynamics, this can generate both procyclical and countercyclical portfolio behaviors.

\footnotetext{
${ }^{8}$ Indeed,

$$
\frac{\gamma \rho}{\sigma_{P}(1-\gamma)} \frac{\partial g(X)}{\partial X}=\frac{\gamma \rho}{\sigma_{P}(1-\gamma)} \frac{\partial g_{0}(X)}{\partial X}+O^{2}(\gamma, \sqrt{\varphi})
$$

${ }^{9}$ The proof is obtained with similar arguments as in Trojani and Vanini (2001a) and is given for completeness.
} 
The impact of a concern for model uncertainty as a function of the underyling state vector $(\kappa, \psi)^{\prime}$ is nonlinear, bounded and depends on the opportunity set state $X$ only. When equity risk barely pays off ( $\kappa$ goes to zero) and agent's sensitivity to stochastic opportunity set fades down $(\psi$ goes to zero), pessimism reduces the ratio between pessimistic and standard myopic portfolio demands. Indeed, when $\kappa$ and $\psi$ are small desired equity holdings in the absence of pessimism are also small, that is risk exposure is small. Pessimism drives to zero such risk exposure in our model. This is a FORA effect. Conversely, when equity risk pays a lot ( $\kappa$ goes to infinity) and/or agent's sensitivity to stochastic opportunity set rockets ( $\psi$ goes to infinity), the relative portfolio impact of introducing a concern for model misspecification goes to zero. That is, myopic and hedging demands converge to their standard expected utility levels.

Effective risk aversion corrections depend on the state $X$ also through the function $|f(X)|$. The impact of a state dependent pessimism on optimal portfolios as a function of the underyling state function $|f(X)|$ is also nonlinear and bounded. Here, the largest relative portfolio corrections are realized when $|f(X)|$ tends to infinity, that is when investors completely loose their faith in the reference belief. In that case, the myopic demand for equity is squeezed down to zero because expected returns on equity become so uncertain that they kill any incentive to myopic speculative behavior. Moreover, in such a circumstance the hedging demand for equity converges to $-\frac{\rho \psi}{\sigma}$. With no faith at all in the reference belief, risk aversion is swamped by model uncertainty so that any trace of $\gamma$ disappears from hedging demand for equity. Since in our framework model misspecification does not affect agent's perception of volatilities and correlations between the state of the economy and tradables, hedging demand for equity remains however alive and kicking even with an unbounded $|f(X)|$.

\section{Pessimistic exchange equilibrium}

We consider pessimistic exchange equilibria in representative agent economies with an investment opportunity set described by the dynamics

$$
\begin{aligned}
d \widehat{B} & =\widehat{r} d t \\
d X & =\zeta d t+\xi d Z^{X}, \\
\frac{d e}{e} & =\alpha_{e} d t+\sigma_{e}\left[\rho_{e} d Z^{X}+\sqrt{1-\rho_{e}^{2}} d Z^{e}\right], \\
\frac{d \widehat{P}+e d t}{\widehat{P}} & =\widehat{\alpha}_{P} d t+\widehat{\sigma}_{P}\left[\widehat{\rho} d Z^{X}+\sqrt{1-\widehat{\rho}^{2}} d Z^{e}\right]
\end{aligned}
$$

Supply of equity is standardized to 1 share, so that the equilibrium price $\widehat{P}$ of the risky asset coincides with the aggregate wealth of the economy. 'Hat' symbols indicate the equilibrium values of the relevant variables.

Investors are homogeneous in their (reference) beliefs, risk preferences and aversions to model uncertainty. They rationally anticipate that today's opportunity set state $X$ and their misconfidence in the reference belief will jointly affect equilibrium equity expected returns $\widehat{\alpha}_{P}$, equity risk $\widehat{\sigma}_{P}$, equity correlations with the opportunity set $\widehat{\rho}$ and the interest rate $\widehat{r}$.

In general equilibrium the function $g$ in the value function expression (4) is now endogenous to the economy. Hence, $g$ (and any term in a direct expansion of $g$ ) depends on both $\gamma$ and $\sqrt{\varphi}$ in equilibrium. In the sequel we start by characterizing the equilibrium function $\widehat{g}$ as the solution of a particular differential equation and compute in a second step the implied equilibrium quantities in terms of $\widehat{g}$. These abstract characterizations already give strong insights into the structure of the implied pessimistic equilibria. We then compute explicitly some perturbative approximations for the relevant equilibrium variables starting from a series expansion for $\widehat{g}$. The next definition introduces the equilibrium concept used in the present and the following sections. 
Definition 3 A pessimistic exchange equilibrium is a vector process $(\widehat{P}, \widehat{r}, \widehat{w}, \widehat{c})^{\prime}$ such that:

- Representative agent's portfolio and consumption rules, $\widehat{w}$ and $\widehat{c}$, are optimal, i.e. they satisfy

$$
\begin{aligned}
\widehat{c} & =\left(\frac{e^{\gamma \widehat{g}}}{\delta}\right)^{\frac{1}{\gamma-1}}, \\
\widehat{w} & =\frac{1}{1-\left(\gamma-\sqrt{\frac{2 \varphi}{\widehat{G}(\widehat{w})}}|f(X)|\right)} \cdot\left(\frac{\widehat{\alpha}_{P}-r}{\widehat{\sigma}_{P}^{2}}+\left(\gamma-\sqrt{\frac{2 \varphi}{G(\widehat{w})}}|f(X)|\right) \frac{\partial g}{\partial X} \frac{\widehat{\rho} \xi}{\widehat{\sigma}_{P}}\right),
\end{aligned}
$$

where

$$
\widehat{G}(w)=\widehat{\sigma}_{P}^{2} w^{2}+\xi^{2}\left(\frac{\partial \widehat{g}}{\partial X}\right)^{2}+2 w \widehat{\rho} \widehat{\sigma}_{P} \xi \frac{\partial \widehat{g}}{\partial X}
$$

- Financial and good markets clear, i.e. $\widehat{w}=1$ and $\widehat{c} \widehat{P}=e$.

The next subsections compute the relevant equilibrium quantities for an abstract pessimistic exchange economy. We will show that to first order classical risk aversion completely determines the equilibrium expected cumulative returns on equity, the volatility of cumulative returns, and their correlation with the opportunity set changes $d X$. On the other hand, pessimism induces to first order basically lower equilibrium interest rates and higher equity risk premia. However, equilibrium consumption and equity returns dynamics are influenced to second order, through the joint interplay of classical risk aversion and (time varying) model uncertainty aversion. Thus, a more accurate analysis of the impact of pessimism on worst case equity premia requires developing higher order perturbative approximations for the implied optimal policies. We compute such higher order approximations below, for some examples of a pessimistic exchange economy.

Part of the first order general equilibrium results in this paper apply readily to any equilibrium model of maxmin consumption/portfolio choice which describes deviations from the reference belief in terms of the relative entropy $\frac{1}{2} h^{\prime} h$. For instance, models including the ones in Anderson, Hansen, and Sargent (2000) and Maenhout (1999) can be solved along similar pertubative lines as the ones used for the present setting, leading to first-order equilibrium stock price dynamics and consumption policies identical to the ones obtained in this paper (see for instance Trojani and Vanini (2001a)). By contrast, first order equilibrium interest rates and risk premia crucially differ from those obtained in this paper. On the other hand, the higher order properties of equilibrium stock price dynamics, optimal consumption, interest rates and equity premia in this paper are different from the ones in the related literature on robust optimal consumption and portfolio selection.

\subsection{Representative agent's equilibrium value function}

It is a remarkable fact that while in partial equilibrium the value function of the pessimistic consumption investment problem cannot be written explicitly as the solution of a single-agent HJB equation, in general equilibrium this is possible. The next theorem characterizes the pessimistic value function of a pessimistic investor via the solution of a particular differential equation.

Theorem 4 In general equilibrium the value function of a pessimistic representative agent is

$$
\widehat{J}(\widehat{Y})=\frac{1}{\delta} \frac{\left(e^{\widehat{g}(X)} \widehat{P}\right)^{\gamma}-1}{\gamma},
$$


where $\widehat{g}$ is the solution to the differential equation

$$
\begin{aligned}
0= & \frac{1}{\gamma}\left(\left(\frac{e^{\gamma g}}{\delta}\right)^{\frac{1}{\gamma-1}}-\delta\right)+\alpha_{e}+\frac{\gamma-1}{2} \sigma_{e}^{2}+\frac{1}{1-\gamma}\left(\zeta+\gamma \xi \rho_{e} \sigma_{e}\right) \frac{\partial g}{\partial X}+\frac{\gamma}{(1-\gamma)^{2}} \frac{\xi^{2}}{2}\left(\frac{\partial g}{\partial X}\right)^{2} \\
& +\frac{1}{1-\gamma} \frac{\xi^{2}}{2} \frac{\partial^{2} g}{\partial^{2} X}-\frac{\sqrt{2 \varphi}}{(1-\gamma)}|f|\left((1-\gamma)^{2} \sigma_{e}^{2}+2(1-\gamma) \rho_{e} \sigma_{e} \xi \frac{\partial g}{\partial X}+\left(\xi \frac{\partial g}{\partial X}\right)^{2}\right)^{\frac{1}{2}} .
\end{aligned}
$$

Notice that the solution $\widehat{g}_{\log , \varphi}$ of (14) for the log utility case $\gamma \rightarrow 0$ solves the differential equation

$$
0=\delta(\ln (\delta)-g)+\alpha_{e}-\frac{\sigma_{e}^{2}}{2}+\zeta \frac{\partial g}{\partial X}+\frac{\xi^{2}}{2} \frac{\partial^{2} g}{\partial^{2} X}-\sqrt{2 \varphi}|f|\left(\sigma_{e}^{2}+2 \rho_{e} \sigma_{e} \xi \frac{\partial g}{\partial X}+\left(\xi \frac{\partial g}{\partial X}\right)^{2}\right)^{\frac{1}{2}},
$$

which is typically easier to solve than (14). Moreover, notice that when setting $\varphi=0$ equation (15) characterizes the value function of a standard (non pessimistic) representative agent economy populated by $\log$ utility investors.

For models where $\widehat{g}_{\log , \varphi}$ can be computed exactly by solving (15), it is possible to give equilibrium asymptotics that only depend on the risk aversion term $\gamma$, rather than on both $\gamma$ and $\sqrt{\varphi}$. On the other hand, if a closed form solution is known only for the special case $\gamma=0, \varphi=0$, it will be necessary to expand in both the risk aversion and the model uncertainty aversion parameters in order to obtain a set of asymptotics for the underlying value function and optimal policies. We give three examples on this topic, two corresponding to a model setting where $\widehat{g}_{\log , \varphi}$ can be given explicitly, and one where only $\widehat{g}_{\log , 0}$ can be computed in closed form. We start with an example that is akin to a general equilibrium extension of the partial equilibrium model in Kim and Omberg (1996), allowing for the existence of intermediate consumption.

Example 5 Ornstein Uhlenbeck state dynamics for expected dividend growth and constant pessimism:

$$
\begin{aligned}
d X & =-\lambda(X-\bar{X}) d t+\xi d Z^{X}, \\
\frac{d e}{e} & =X d t+\sigma_{e}\left[\rho_{e} d Z^{X}+\sqrt{1-\rho_{e}^{2}} d Z^{e}\right], \\
\frac{d \widehat{P}+e d t}{\widehat{P}} & =\widehat{\alpha}_{P} d t+\widehat{\sigma}_{P}\left[\widehat{\rho} d Z^{X}+\sqrt{1-\widehat{\rho}^{2}} d Z^{e}\right], \\
f(X) & =1 .
\end{aligned}
$$

where $\lambda, \bar{X}, \xi, \sigma_{e}>0$ and $\rho_{e} \in[-1,1]$. The differential equation (15) reads explicitly

$0=\delta(\ln (\delta)-g)+X-\frac{\sigma_{e}^{2}}{2}-\lambda(X-\bar{X}) \frac{\partial g}{\partial X}+\frac{\xi^{2}}{2} \frac{\partial^{2} g}{\partial^{2} X}-\sqrt{2 \varphi}\left(\sigma_{e}^{2}+2 \rho_{e} \sigma_{e} \xi \frac{\partial g}{\partial X}+\left(\xi \frac{\partial g}{\partial X}\right)^{2}\right)^{\frac{1}{2}}$,

with solution

$$
\widehat{g}_{\log , \varphi}(X)=a(\varphi)+X
$$

where

$$
\begin{aligned}
a(\varphi) & =\ln (\delta)-\frac{\sigma_{e}^{2}}{2 \delta}+\frac{\lambda \bar{X}}{\delta(\lambda+\delta)}-\frac{\sqrt{2 \varphi}}{\delta}\left(\sigma_{e}^{2}+\frac{2 \rho_{e} \sigma_{e} \xi}{\lambda+\delta}+\left(\frac{\xi}{\lambda+\delta}\right)^{2}\right)^{\frac{1}{2}} \\
b & =\frac{1}{\lambda+\delta}
\end{aligned}
$$


In this model setting pessimism affects the equilibrium function $\widehat{g}_{\log , \varphi}$ by a translation that leaves $\widehat{g}_{\log , \varphi}$ 's derivative unchanged, when comparing with the non pessimistic model setting implied by $\varphi=0$. This fact has consequences for the kind of equilibrium impact that pessimism has on the dynamics of asset prices and on equity premia (cf. the next two sections). In the next example, $\widehat{g}_{\log , \varphi}$ 's derivatives are affected by pessimism in equilibrium.

Example 6 Cox Ingersoll Ross state dynamics for dividend volatility and pessimistic maximal distrust function proportional to dividend volatilities

$$
\begin{aligned}
d X & =-\lambda(X-\bar{X}) d t+\xi \sqrt{X} d Z^{X} \\
\frac{d e}{e} & =\alpha_{e} d t+\sigma_{e} \sqrt{X}\left[\rho_{e} d Z^{X}+\sqrt{1-\rho_{e}^{2}} d Z^{e}\right] \\
\frac{d \widehat{P}+e d t}{\widehat{P}} & =\widehat{\alpha}_{P} d t+\widehat{\sigma}_{P}\left[\widehat{\rho} d Z^{X}+\sqrt{1-\widehat{\rho}^{2}} d Z^{e}\right] \\
f(X) & =\frac{\sqrt{X}}{\sqrt{X}} .
\end{aligned}
$$

where $\lambda, \bar{X}, \xi, \alpha_{e}, \sigma_{e}>0$ and $\rho_{e} \in[-1,1]$. The differential equation (15) reads explicitly

$$
\begin{aligned}
0= & \delta(\ln (\delta)-g)+\alpha_{e}-\frac{\sigma_{e}^{2}}{2} X-\lambda(X-\bar{X}) \frac{\partial g}{\partial X}+\frac{\xi^{2}}{2} X \frac{\partial^{2} g}{\partial^{2} X} \\
& -\sqrt{\frac{2 \varphi}{\bar{X}}} X\left(\sigma_{e}^{2}+2 \rho_{e} \sigma_{e} \xi \frac{\partial g}{\partial X}+\left(\xi \frac{\partial g}{\partial X}\right)^{2}\right)^{\frac{1}{2}},
\end{aligned}
$$

with solution

$$
\widehat{g}_{\log , \varphi}(X)=a(\varphi)+b(\varphi) X,
$$

where

$$
a(\varphi)=\ln \delta+\frac{\alpha_{e}}{\delta}+\frac{\lambda \bar{X} b(\varphi)}{\delta},
$$

and $b=b(\varphi)$ is a root of the quadratic equation

$$
b^{2}\left((\delta+\lambda)^{2}-\frac{2 \varphi \xi^{2}}{\bar{X}}\right)+\sigma_{e}\left((\delta+\lambda) \sigma_{e}-\frac{4 \varphi \rho_{e} \xi}{\bar{X}}\right) b+\sigma_{e}^{2}\left(\frac{\sigma_{e}^{2}}{4}-\frac{2 \varphi}{\bar{X}}\right)=0
$$

such that

$$
b(\varphi) \leq b(0)=-\frac{\sigma_{e}^{2}}{2(\lambda+\delta)} .
$$

In general, it will not be possible to solve the differential equation (14) in the log utility case (15), even when a solution for the special case $\varphi=0$ is available. An example of such a situation is the following.

Example 7 Geometric Ornstein Uhlembeck state dynamics for dividend volatilities

$$
\begin{aligned}
d X & =-\lambda(X-\bar{X}) d t+\xi X d Z^{X} \\
\frac{d e}{e} & =\alpha_{e} d t+\sigma_{e} X\left[\rho_{e} d Z^{X}+\sqrt{1-\rho_{e}^{2}} d Z^{e}\right], \\
\frac{d \widehat{P}+e d t}{\widehat{P}} & =\widehat{\alpha}_{P} d t+\widehat{\sigma}_{P}\left[\widehat{\rho} d Z^{X}+\sqrt{1-\widehat{\rho}^{2}} d Z^{e}\right], \\
f_{1}(X) & =1 ; \quad f_{2}(X)=\frac{X}{\bar{X}}
\end{aligned}
$$


where $\lambda, \bar{X}, \xi, \alpha_{e}, \sigma_{e}>0$ and $\rho_{e} \in[-1,1]$. The differential equation (15) reads explicitly

$$
\begin{aligned}
0= & \delta(\ln (\delta)-g)+\alpha_{e}-\frac{\sigma_{e}^{2}}{2} X^{2}-\lambda(X-\bar{X}) \frac{\partial g}{\partial X}+\frac{\xi^{2}}{2} X^{2} \frac{\partial^{2} g}{\partial^{2} X} \\
& -\sqrt{2 \varphi} X f_{i}(X)\left(\sigma_{e}^{2}+2 \rho_{e} \sigma_{e} \xi \frac{\partial g}{\partial X}+\left(\xi \frac{\partial g}{\partial X}\right)^{2}\right)^{\frac{1}{2}} .
\end{aligned}
$$

For this equation no closed form solution exists when $\varphi>0$ even if for $\varphi=0$ we have

$$
\widehat{g}_{\log , 0}(X)=a_{0}+b_{0} X+c_{0} X^{2},
$$

where

$$
\begin{aligned}
& a_{0}=\ln (\delta)+\frac{\alpha_{e}}{\delta}+\frac{\lambda \bar{X}}{\delta} b_{0} \\
& b_{0}=\frac{2 \lambda \bar{X}}{(\delta+\delta)} c_{0} \\
& c_{0}=-\frac{\sigma_{e}^{2}}{2\left(2 \lambda+\delta-\xi^{2}\right)} .
\end{aligned}
$$

When, as in Example 7, closed form solutions are available only for the special case $\gamma=0, \varphi=0$, it is possible to develop perturbative approximations that expand $\widehat{g}$ with respect to $\gamma$ and $\sqrt{\varphi}$ in a neighborhood of an economy populated by logarithmic, non pessimistic, investors $(\gamma=0, \varphi=0)$. When, as in Example 5 and 6, closed form solutions for the case $\gamma=0$ are available, it is possible to expand $\widehat{g}$ only with respect to $\gamma$ in a neighborhhod of an economy populated by logarithmic pessimistic investors $(\gamma=0, \varphi>0)$. Depending, on the given explicit model setting the first or the second of these perturbation approaches can be adopted. Notice, that for $\gamma \neq 0$ and $\varphi=0$ no closed form solution is generally available in the presence of state dependent opportunity sets.

\subsection{Equilibrium cumulative returns on equity}

The proof of Theorem 14 already implies the equilibrium dynamics of pessimistic cumulative returns on equity, in dependence of the equilibrium function $\widehat{g}$. This is the next proposition.

Proposition 8 The pessimistic exchange equilibrium implies conditional expectations of cumulative returns on equity, conditional variances and correlations given by

$$
\begin{aligned}
\widehat{\alpha}_{P} & =\alpha_{e}+\frac{\gamma}{1-\gamma}\left(\frac{\partial \widehat{g}}{\partial X}\left(\zeta+\xi \rho_{e} \sigma_{e}\right)+\frac{\xi^{2}}{2}\left(\frac{\gamma}{1-\gamma}\left(\frac{\partial \widehat{g}}{\partial X}\right)^{2}+\frac{\partial^{2} \widehat{g}}{\partial^{2} X}\right)\right)+\left(\frac{e^{\gamma \widehat{g}}}{\delta}\right)^{\frac{1}{\gamma-1}} \\
\widehat{\sigma}_{P}^{2} & =\sigma_{e}^{2}+\frac{\gamma}{1-\gamma} 2 \rho_{e} \sigma_{e} \xi \frac{\partial \widehat{g}}{\partial X}+\left(\frac{\gamma}{1-\gamma} \xi\right)^{2}\left(\frac{\partial \widehat{g}}{\partial X}\right)^{2}, \\
\widehat{\rho} & =\frac{\sigma_{e} \rho_{e}+\frac{\gamma}{1-\gamma} \frac{\partial \widehat{g}}{\partial X} \xi}{\sqrt{\sigma_{e}^{2}+\frac{\gamma}{1-\gamma} 2 \rho_{e} \sigma_{e} \xi \frac{\partial \widehat{g}}{\partial X}+\left(\frac{\gamma}{1-\gamma} \xi\right)^{2}\left(\frac{\partial \widehat{g}}{\partial X}\right)^{2}}}
\end{aligned}
$$

respectively.

Several remarks can be drawn from Proposition 8. First of all, notice that in the log utility case we have for any $\varphi \geq 0$

$$
\widehat{\alpha}_{P}=\alpha_{e} \quad, \quad \widehat{\sigma}_{P}^{2}=\sigma_{e}^{2} \quad, \quad \widehat{\rho}=\rho_{e} .
$$


Thus, pessimism has no impact on the equilibrium dynamics of risky assets returns in this case. As a consequence, we see that pessimism can affect equilibrium equity expected returns, volatilites and correlations, only through the joint interplay of risk aversion (when $\gamma \neq 0$ ) and model uncertainty aversion (when $\varphi>0$ ). Hence, in order to analyze the interaction of risk aversion and pessimism in determining asset prices one will have to expand $\widehat{g}$ at least to first order, i.e.

$$
\begin{aligned}
\widehat{g} & =\widehat{g}_{\log , 0}+\gamma \widehat{g}_{1}+\sqrt{\varphi} \widehat{g}_{2}+O^{2}(\gamma, \sqrt{\varphi}), \\
\widehat{g} & =\widehat{g}_{\log , \varphi}+\gamma \widehat{g}_{1}+O\left(\gamma^{2}\right),
\end{aligned}
$$

where $\widehat{g}_{\log , 0}\left(\widehat{g}_{\log , \varphi}\right)$ is the solution of the differential equation (15) for the case where a closed expression exists for $\varphi=0$ (for the more general setting $\varphi>0$ ). Furthermore, the functions $\widehat{g}_{1}$ and $\widehat{g}_{2}$ will have to be determined. Indeed, since pessimism acts indirectly on equity returns dynamics by affecting only the equilibrium function $\widehat{g}$ and its derivatives, the impact of pessimism on equity returns is of an order no less than two with respect to both the risky aversion and the model uncertainty aversion parameters $\gamma, \sqrt{\varphi}$. Neglecting this fact yields in the next corollary a set of immediate $O^{2}(\gamma, \varphi)-$ order equilibrium approximations, which however only reflect the direct impact of standard risk aversion on equilibrium asset prices $^{10}$.

Corollary 9 The pessimistic exchange equilibrium implies conditional expectations of cumulative returns on equity, conditional volatilities and correlations given by

$$
\begin{aligned}
& \widehat{\alpha}_{P} \stackrel{\circ}{=} \alpha_{e}+\frac{\gamma}{1-\gamma}\left(\frac{\partial \widehat{g}_{\log , 0}}{\partial X}\left(\zeta+\xi \rho_{e} \sigma_{e}\right)+\frac{\xi^{2}}{2} \frac{\partial^{2} \widehat{g}_{\log , 0}}{\partial^{2} X}\right)+\alpha_{e}+\delta\left(1-\gamma\left(\widehat{g}_{\log , 0}-\ln (\delta)\right)\right), \\
& \widehat{\sigma}_{P} \stackrel{\circ}{\sigma_{e}^{2}+\frac{\gamma}{1-\gamma} 2 \rho_{e} \sigma_{e} \xi \frac{\partial \widehat{g}_{\log , 0}}{\partial X}+\left(\frac{\gamma}{1-\gamma} \xi \frac{\partial \widehat{g}_{\log , 0}}{\partial X}\right)^{2}} \text {, } \\
& \widehat{\rho} \stackrel{\circ}{=} \frac{\sigma_{e} \rho_{e}+\gamma \xi \frac{\partial \widehat{g}_{\log , 0}}{\partial X}}{\sqrt{\sigma_{e}^{2}+\frac{\gamma}{1-\gamma} 2 \rho_{e} \sigma_{e} \xi \frac{\partial \widehat{g}_{\log , 0}}{\partial X}+\left(\frac{\gamma}{1-\gamma} \xi \frac{\partial \widehat{g}_{\log , 0}}{\partial X}\right)^{2}}}
\end{aligned}
$$

The observed levels of equity prices, expected returns, volatilities and correlations in an economy with pessimism are observationally equivalent (up to $O^{2}(\gamma, \varphi)$-order) to the ones in a corresponding standard economy. This result is determined by two features of our equilibrium asymptotics. First, pessimism does not affect to first order optimal consumption in the economy (cf. also the partial equilibrium results in Section 3). Second, the financial market clearing condition imposes a fixed equilibrium equity allocation $w=1$ in homogenous economies. This second constraint eliminates the impact of pessimism from the equilibrium aggregate wealth dynamics, even if the optimal investment policies of a pessimistic investor are significantly affected by the relevant degree of pessimism (cf. again Section 3$)^{11}$. These two features of a $O^{2}(\gamma, \varphi)$-order approximation of the equity dynamics have implications for a first order analysis of the way in which pessimism impacts equilibrium equity premia. Indeed, since equity returns are unchanged to first order, pessimism then affects $O^{2}(\gamma, \varphi)$-order risk premia in the reference model by fully impacting only the implied equilibrium interest rates. Moreover, as the next section shows, a pure $O^{2}(\gamma, \varphi)$-order analysis has implications for a discussion of how the implied worst case equity premia in the corresponding worst case scenario are affected by pessimism, since worst case premia are, to $O^{2}(\gamma, \varphi)$-order, fully determined by standard risk aversion. The next section analyzes the impact of pessimism on equilibrium equity premia.

\footnotetext{
${ }^{10}$ In the sequel we use the symbol $\triangleq$ to denote equality up to terms of order $O^{2}(\gamma, \sqrt{\varphi})$.

${ }^{11}$ In heterogenous agents economies pessimism will also affect the aggregate wealth dynamics also to first order; see Trojani and Vanini (2001a).
} 


\subsection{Equilibrium equity premia and interest rates}

Knowledge of the equilibrium equity dynamics in Corollary 9 allows the explicit computation of the implied worst case drift contamination and the corresponding reference model and worst case model equity premia. Determining analytically also the worst case equity premium is economically important, in order to assess the plausibility of the worst case scenario implied by a pessimistic exchange economy.

Corollary 10 The pessimistic worst case model and reference model equilibrium equity premia are given by

$$
\left(\widehat{\alpha}_{P}-r\right)_{\widehat{h}^{*}}=\widehat{\alpha}_{P}-\widehat{r}+\widehat{\sigma}_{P}^{2}\left[\widehat{\rho} \widehat{h}^{X *}+\sqrt{1-\widehat{\rho}^{2}} \widehat{h}^{P *}\right]=\widehat{\sigma}_{P}^{2}-\gamma\left(\widehat{\sigma}_{P}^{2}+\widehat{\rho} \xi \widehat{\sigma}_{P} \frac{\partial \widehat{g}}{\partial X}\right)
$$

and

$$
\widehat{\alpha}_{P}-\widehat{r}=\left(\widehat{\alpha}_{P}-r\right)_{\widehat{h}^{*}}+\sqrt{\frac{2 \varphi}{\sigma_{e}^{2}+\frac{2 \rho_{e} \sigma_{e} \xi}{1-\gamma} \frac{\partial \widehat{g}}{\partial X}+\left(\frac{\xi}{1-\gamma} \frac{\partial \widehat{g}}{\partial X}\right)^{2}}}|f(X)|\left(\widehat{\sigma}_{P}^{2}+\widehat{\rho} \xi \widehat{\sigma}_{P} \frac{\partial \widehat{g}}{\partial X}\right)
$$

respectively.

From Corollary 10 we notice that reference model equity premia are directly affected by time varying pessimism. They are higher in the presence of model uncertainty if and only if

$$
\widehat{\sigma}_{P}^{2}+\widehat{\rho} \xi \widehat{\sigma}_{P} \frac{\partial \widehat{g}}{\partial X}>0
$$

This requirement is the condition that the sum of the standard and pessimistic speculative demands for equity dominates over the corresponding intertemporal hedging demand.

On the other hand, Corollary 10 also tells us that also worst case model equity premia are affected only indirectly by pessimism, through the altered equilibrium function $\widehat{g}$ and the implied equilibrium volatilities and correlations $\widehat{\sigma}_{P}$ and $\widehat{\rho}$. For instance, in the logarithmic utility case we have (using also results in Corollary 9)

$$
\left(\widehat{\alpha}_{P}-r\right)_{\widehat{h}^{*}}=\widehat{\sigma}_{e}^{2}
$$

that is, worst case premia are precisely the equity premia of a corresponding standard (non pessimistic) economy. Hence, we see that - similarly to the equilibrium equity dynamics - it is the joint interplay of risk aversion (when $\gamma \neq 0$ ) and model uncertainty aversion (when $\varphi>0$ ) that determines equilibrium equity premia in the wost case. In the same vain as for the results in Corollary 9 this implies that pure $O^{2}(\gamma, \varphi)$-order asymptotics are not sufficient to fully understand the impact of pessimism on worst case equity premia. Indeed, as the next straightforward corollary states, up to $O^{2}(\gamma, \varphi)-$ order worst case premia are completely determined by risk aversion.

Corollary 11 The pessimistic worst case model and reference model equilibrium equity premia are given by

$$
\begin{aligned}
\left(\widehat{\alpha}_{P}-\widehat{r}\right)_{h *} & \stackrel{\circ}{=} \sigma_{e}^{2}\left(1+\gamma 2 \frac{\rho_{e}}{\sigma_{e}} \xi \frac{\partial \widehat{g}_{\log , 0}}{\partial X}\right)-\gamma\left(\sigma_{e}^{2}+\rho_{e} \sigma_{e} \xi \frac{\partial \widehat{g}_{\log , 0}}{\partial X}\right) \\
& =\sigma_{e}^{2}(1-\gamma)+\gamma \rho_{e} \xi \frac{\partial \widehat{g}_{\log , 0}}{\partial X}
\end{aligned}
$$

and

$$
\widehat{\alpha}_{P}-\widehat{r} \stackrel{\circ}{=} \sigma_{e}^{2}(1-2 \gamma)+\sqrt{\frac{2 \varphi}{\sigma_{e}^{2}+\left(\xi \frac{\partial \widehat{g}_{0, \log }}{\partial X}\right)^{2}+2 \rho_{e} \sigma_{e} \xi \frac{\partial \widehat{g}_{0, \log }}{\partial X}}}|f(X)|\left(\sigma_{e}^{2}+\rho_{e} \sigma_{e} \frac{\partial \widehat{g}_{\log , 0}}{\partial X}\right),
$$

respectively. 
Since up to $O^{2}(\gamma, \varphi)$-errors the equilibrium reference belief $\widehat{\alpha}_{P}$ on conditional expected cumulative returns is unaffected by the degree of pessimism, the first order effect of an aversion to model uncertainty fully acts on the equilibrium riskless rate $\widehat{r}$ - which under condition (39) is lower that in the absence of pessimism - inducing higher equity premia. Basically, to $O^{2}(\gamma, \varphi)$-order uncertainty on expected equity returns triggers a pure asset substitution effect, which in equilibrium causes pessimistic investors to allocate a different fraction of their aggregate saving to discount bonds. However, to $O^{2}(\gamma, \varphi)$-order pessimism does not trigger additional precautionary savings as it leaves untouched the equilibrium fraction of aggregate consumption on aggregate wealth.

As expected, $O^{2}(\gamma, \varphi)$-order worst case equity premia in Corollary 11 are completely determined by standard risk aversion. Hence, worst case equity premia equal the standard CRRA equity premia, implying that equilibrium interest rates in the presence of pessimism adjust in a way that exactly offsets the conservative change in expected returns implied by the associated worst case scenario. It is again the homogenous structure of our exchange economy that implies such a restriction on worst case equity premia. Indeed, the financial market clearing condition for our pessimistic investor can be expressed equivalently in terms of the optimal investment policy with respect either to the reference model or to the worst case scenario. In the reference model, a state-dependent correction of effective risk aversion arises (cf. Equation (6)). In the worst case model, investors compute standard CRRA optimal policies using worst case expected returns. This yields a financial market clearing condition given by

$$
(1-\gamma) \widehat{\sigma}_{P}^{2}=\left(\widehat{\alpha}_{P}-\widehat{r}\right)_{h^{*}}+\gamma \widehat{\rho} \widehat{\sigma}_{P} \widehat{\psi}_{\log },
$$

which implies precisely the worst case premium in Corollary 11. Since to $O^{2}(\gamma, \varphi)$-order $\widehat{\sigma}_{P}^{2}$ and $\widehat{\rho}$ are by Corollary 9 completely determined by standard risk aversion, the first order market clearing effect on equilibrium interest rates and the worst case adjustment in expected returns on equity have to match exactly, inducing worst case equity premia that do not depend on the degree of pessimism in the economy. Therefore, in Section 5 below where we discuss some explicit models we will make use of $O^{2}(\gamma, \varphi)$-order equilibrium asymptotics exclusively to investigate and quantify the direct impact of pessimism on equity premia and interest rates in models when no higher order asymptotic solutions can be provided. The general caracterization of such higher order asymptotics is provided in the next section.

\subsection{Higher order properties of pessimistic value functions}

To analyze the equilibrium impact of an interplay between risk aversion and pessimism, it is necessary to compute equilibrium asymptotics that take into account (at least) second order effects in $\gamma, \sqrt{\varphi}$, or in both perturbation parameters. This can be achieved (cf. for instance Proposition 8 and 10) by characterizing the function $\widehat{g}$ (at least) to first order in $\gamma$ and $\sqrt{\varphi}$. Hence, depending on the model under scrutiny (cf. for instance the examples in the last section), we can expand $\widehat{g}$ to first order either only with respect to $\gamma$ or with respect to both $\gamma$ and $\sqrt{\varphi}$. This gives

$$
\begin{aligned}
& \widehat{g}=\widehat{g}_{\log , \varphi}+\gamma \widehat{g}_{1}+O\left(\gamma^{2}\right) \\
& \widehat{g}=\widehat{g}_{\log , 0}+\gamma \widehat{g}_{1}+\sqrt{\varphi} \widehat{g}_{2}+O^{2}(\gamma, \sqrt{\varphi})
\end{aligned}
$$

respectively. Expansions of the form (42) will provide by Proposition 8 and 10 a set of $O\left(\gamma^{3}\right)$-equilibrium asymptotics, once $\widehat{g}_{1}$ in (42) has been determined. On the other hand, expansions of the form (43) can provide a set of $O^{3}(\gamma, \sqrt{\varphi})$-equilibrium asymptotics ${ }^{12}$. In this case, both $\widehat{g}_{1}$ and $\widehat{g}_{2}$ will have to be determined. Therefore, the issue is now to characterize $\widehat{g}_{1}$ and $\widehat{g}_{2}$, in dependence of the relevant zeroth order term $\widehat{g}_{\log , 0}$ (or $\widehat{g}_{\log , \varphi}$ ). This is the next proposition.

\footnotetext{
${ }^{12}$ We used the symbol $O^{3}(\gamma, \sqrt{\varphi})$ as a short notation for $O\left(\|\gamma, \sqrt{\varphi}\|^{3}\right)$.
} 
Proposition 12 The first order functions $\widehat{g}_{1}, \widehat{g}_{2}$ in (43) are solutions of the differential equations

$$
\begin{aligned}
& 0=\mathbf{D}_{0}\left(\widehat{g}_{1}\right)+\mathbf{R}_{1}\left(\widehat{g}_{\log , 0}\right), \\
& 0=\mathbf{D}_{0}\left(\widehat{g}_{2}\right)+\mathbf{R}_{2}\left(\widehat{g}_{\log , 0}\right),
\end{aligned}
$$

where the differential operators $\mathbf{D}_{0}, \mathbf{R}_{1}, \mathbf{R}_{2}$ are defined by

$$
\begin{aligned}
\mathbf{D}_{0}(g)= & -\delta g+\zeta \frac{\partial g}{\partial X}+\frac{\xi^{2}}{2} \frac{\partial^{2} g}{\partial^{2} X} \\
\mathbf{R}_{1}(g)= & \delta\left(\left(\frac{g}{2}-1-\ln (\delta)\right) g+\ln (\delta)\left(1+\frac{\ln (\delta)}{2}\right)\right)+\frac{\sigma_{e}^{2}}{2} \\
& +\left(\zeta+\xi \rho_{e} \sigma_{e}\right) \frac{\partial g}{\partial X}+\frac{\xi^{2}}{2}\left(\frac{\partial g}{\partial X}\right)^{2}+\frac{\xi^{2}}{2} \frac{\partial^{2} g}{\partial^{2} X}, \\
\mathbf{R}_{2}(g)= & -\sqrt{2}|f|\left(\sigma_{e}^{2}+2 \rho_{e} \sigma_{e} \xi \frac{\partial g}{\partial X}+\left(\xi \frac{\partial g}{\partial X}\right)^{2}\right)^{\frac{1}{2}} .
\end{aligned}
$$

The first order function $\widehat{g}_{1}$ in (42) is solution of the differential equation

$$
0=\mathbf{D}_{1}\left(\widehat{g}_{\log , \varphi}, \widehat{g}_{1}\right)+\mathbf{R}_{1}\left(\widehat{g}_{\log , \varphi}\right),
$$

where

$$
\mathbf{D}_{1}(g, q)=\mathbf{D}_{0}(q)-\sqrt{2 \varphi}|f| \frac{\xi \frac{\partial g}{\partial X}+\rho_{e} \sigma_{e}}{\left(\sigma_{e}^{2}+2 \rho_{e} \sigma_{e} \xi \frac{\partial g}{\partial X}+\left(\xi \frac{\partial g}{\partial X}\right)^{2}\right)^{\frac{1}{2}}} \xi \frac{\partial q}{\partial X}
$$

From Proposition 12, $\widehat{g}_{1}, \widehat{g}_{2}$, are solutions of a linear second order differential equation with the same homogenous equation $\mathbf{D}_{0}(g)=0$ and inhomogeneities $\mathbf{R}_{1}\left(\widehat{g}_{\log , 0}\right)$ and $\mathbf{R}_{2}\left(\widehat{g}_{\log , 0}\right)$, respectively. Similarly, in a $O\left(\gamma^{2}\right)$-expansion for $\widehat{g}$ the first order term $\widehat{g}_{1}$ is a solution of a second order linear differential equation with homogenous equation $\mathbf{D}_{1}\left(\widehat{g}_{\log , \varphi}, g\right)=0$ and inhomogeneity $\mathbf{R}_{1}\left(\widehat{g}_{\log , \varphi}\right)$. Given a system of fundamental solutions to the homogenous equation $\mathbf{D}_{0}(g)=0$, (or $\mathbf{D}_{1}\left(\widehat{g}_{\log , \varphi}, g\right)=$ $0) \widehat{g}_{1}$ and $\widehat{g}_{2}$ can be in principle computed by the variation of constant method ${ }^{13}$. However, notice that for some interesting model settings a polynomial guess already gives the solution. We now introduce some examples in this respect that will be useful later on to analyze and quantify the higher order impact of pessimism on asset prices and worst case equity premia in some specific models. We start again from a model with mean reverting expected dividend growth and constant dividend volatilities.

Corollary 13 With the explicit model dynamics of Example 5 the function $\widehat{g}_{1}$ in (42) is the solution of the differential equation

$$
0=\mathbf{D}_{1}\left(\widehat{g}_{1}\right)+\mathbf{R}_{1}(X)
$$

where

$$
\begin{aligned}
\mathbf{D}_{1}(g)= & -\delta g-\left(\lambda(X-\bar{X})+\sqrt{2 \varphi} \xi \frac{\xi b+\rho_{e} \sigma_{e}}{\left(\sigma_{e}^{2}+2 \rho_{e} \sigma_{e} \xi b+(\xi b)^{2}\right)^{\frac{1}{2}}}\right) \frac{\partial g}{\partial X}+\frac{\xi^{2}}{2} \frac{\partial^{2} g}{\partial^{2} X} \\
\mathbf{R}_{1}(X)= & \delta\left(\left(\frac{a(\varphi)+b X}{2}-1-\ln (\delta)\right)(a(\varphi)+b X)+\ln (\delta)\left(1+\frac{\ln (\delta)}{2}\right)\right) \\
& +\left(\lambda(\bar{X}-X)+\xi \rho_{e} \sigma_{e}\right) b+\frac{\sigma_{e}^{2}+(\xi b)^{2}}{2}
\end{aligned}
$$

\footnotetext{
13 This, however, will often imply rather complicated expressions for a solution, since the fundamental system associated with quite standard state dynamics like a CIR one is already given by two non trivial basis functions. Moreover, the final solution will contain linear combinations of integrals of these functions.
} 
and $a(\varphi)$ and $b$ are given in (22) and (23), respectively. Therefore $\widehat{g}_{1}$ is given by

$$
\widehat{g}_{1}(X)=\alpha(\varphi)+\beta(\varphi) X+\varepsilon X^{2},
$$

where

$$
\begin{aligned}
\varepsilon= & \frac{\delta b^{2}}{2(2 \lambda+\delta)} \\
\beta(\varphi)= & \frac{2}{\lambda+\delta}\left(\lambda \bar{X}-\sqrt{2 \varphi} \xi \frac{\xi b+\rho_{e} \sigma_{e}}{\left(\sigma_{e}^{2}+2 \rho_{e} \sigma_{e} \xi b+(\xi b)^{2}\right)^{\frac{1}{2}}}\right) \varepsilon+\frac{(\delta(a(\varphi)-(1+\ln \delta))-\lambda) b}{\lambda+\delta} \\
\alpha(\varphi)= & \frac{\xi^{2} \varepsilon}{\delta}+\frac{1}{\delta}\left(\lambda \bar{X}-\sqrt{2 \varphi} \xi \frac{\xi b+\rho_{e} \sigma_{e}}{\left(\sigma_{e}^{2}+2 \rho_{e} \sigma_{e} \xi b+(\xi b)^{2}\right)^{\frac{1}{2}}}\right) \beta(\varphi) \\
& +\left(\frac{a(\varphi)}{2}-1\right) a(\varphi)+(\ln \delta)\left(1+\frac{1}{2} \ln \delta-a(\varphi)\right)+\frac{b}{\delta}\left(\lambda \bar{X}+\xi \rho_{e} \sigma_{e}\right)+\frac{\xi^{2} b^{2}+\sigma_{e}^{2}}{2 \delta}
\end{aligned}
$$

The second example considers again the solution of a model with heteroschedastic returns and a maximal distrust function proportional to the conditional dividend volatilities.

Corollary 14 With the explicit model dynamics of Example 6 the function $\widehat{g}_{1}$ in (42) is the solution of the differential equation

$$
0=\mathbf{D}_{1}\left(\widehat{g}_{1}\right)+\mathbf{R}_{1}(X)
$$

where

$$
\begin{aligned}
\mathbf{D}_{1}(g)= & -\delta g-\left(\lambda(X-\bar{X})+\sqrt{2 \varphi} \xi \frac{\xi b(\varphi)+\rho_{e} \sigma_{e}}{\left(\sigma_{e}^{2}+2 \rho_{e} \sigma_{e} \xi b(\varphi)+(\xi b(\varphi))^{2}\right)^{\frac{1}{2}}} X\right) \frac{\partial g}{\partial X}+\frac{\xi^{2}}{2} X \frac{\partial^{2} g}{\partial^{2} X}, \\
\mathbf{R}_{1}(X)= & \delta\left(\left(\frac{a(\varphi)+b(\varphi) X}{2}-1-\ln (\delta)\right)(a(\varphi)+b(\varphi) X)+\ln (\delta)\left(1+\frac{\ln (\delta)}{2}\right)\right) \\
& +\left(\lambda(\bar{X}-X)+\xi \rho_{e} \sigma_{e} X\right) b(\varphi)+\frac{\sigma_{e}^{2}+(\xi b(\varphi))^{2}}{2} X
\end{aligned}
$$

and $a(\varphi)$ and $b(\varphi)$ are given in (30) and (31), respectively. Therefore $\widehat{g}_{1}$ is given by

$$
\widehat{g}_{1}(X)=\alpha(\varphi)+\beta(\varphi) X+\varepsilon(\varphi) X^{2} \quad,
$$

where

$$
\begin{aligned}
\varepsilon(\varphi)= & \frac{\delta b(\varphi)^{2}}{2\left(\delta+2\left(\lambda+\sqrt{2 \varphi} \frac{\xi}{\sqrt{\bar{X}}} \frac{\xi b(\varphi)+\rho_{e} \sigma_{e}}{\left(\sigma_{e}^{2}+2 \rho_{e} \sigma_{e} \xi b(\varphi)+(\xi b(\varphi))^{2}\right)^{\frac{1}{2}}}\right)\right)}, \\
\beta(\varphi)= & \frac{2 \lambda \bar{X}+\xi^{2}}{\lambda+\delta+\sqrt{2 \varphi} \frac{\xi}{\sqrt{\bar{X}}} \frac{\xi b(\varphi)+\rho_{e} \sigma_{e}}{\left(\sigma_{e}^{2}+2 \rho_{e} \sigma_{e} \xi b(\varphi)+(\xi b(\varphi))^{2}\right)^{\frac{1}{2}}}} \varepsilon(\varphi) \\
& +\frac{\delta(a(\varphi)-1-\ln \delta) b(\varphi)+\left(\xi \rho_{e} \sigma_{e}-\lambda\right) b(\varphi)+\frac{1}{2}\left(\sigma_{e}^{2}+\xi^{2} b(\varphi)^{2}\right)}{\lambda+\delta+\sqrt{2 \varphi} \frac{\xi}{\sqrt{\bar{X}}} \frac{\xi b(\varphi)+\rho_{e} \sigma_{e}}{\left(\sigma_{e}^{2}+2 \rho_{e} \sigma_{e} \xi b(\varphi)+(\xi b(\varphi))^{2}\right)^{\frac{1}{2}}}}, \\
\alpha(\varphi)= & \frac{\lambda \bar{X}}{\delta} \beta(\varphi)+\frac{\lambda \bar{X}}{\delta} b(\varphi)+\left(\frac{a(\varphi)}{2}-1\right) a(\varphi)+\ln \delta\left(1+\frac{1}{2} \ln \delta-a(\varphi)\right) .
\end{aligned}
$$


The last example considers a model with heteroschedastic expected dividend growth and a constant maximal distrust function $f^{14}$. We first give the closed form expression of $\widehat{g}$ for the case of a $\log$ utility investor.

Example 15 Geometric Ornstein Uhlembeck state dynamics for expected dividend growth and dividend growth volatility:

$$
\begin{aligned}
d X & =-\lambda(X-\bar{X}) d t+\xi X d Z^{X}, \\
\frac{d e}{e} & =\left(X+\frac{\sigma_{e}^{2}}{2} X^{2}\right) d t+\sigma_{e} X\left[\rho_{e} d Z^{X}+\sqrt{1-\rho_{e}^{2}} d Z^{e}\right], \\
\frac{d \widehat{P}+e d t}{\widehat{P}} & =\widehat{\alpha}_{P} d t+\widehat{\sigma}_{P}\left[\widehat{\rho} d Z^{X}+\sqrt{1-\widehat{\rho}^{2}} d Z^{e}\right], \\
f(X) & =1,
\end{aligned}
$$

where $\lambda, \bar{X}, \xi, \sigma_{e}>0$ and $\rho_{e} \in[-1,1]$. The differential equation (15) reads explicitly

$0=\delta(\ln (\delta)-g)+X-\lambda(X-\bar{X}) \frac{\partial g}{\partial X}+\frac{\xi^{2}}{2} X^{2} \frac{\partial^{2} g}{\partial^{2} X}-\sqrt{2 \varphi} X\left(\sigma_{e}^{2}+2 \rho_{e} \sigma_{e} \xi \frac{\partial g}{\partial X}+\left(\xi \frac{\partial g}{\partial X}\right)^{2}\right)^{\frac{1}{2}}$

with solution

$$
\widehat{g}_{\log , \varphi}=a(\varphi)+b(\varphi) X \quad,
$$

where

$$
a(\varphi)=\frac{\lambda \bar{X} b(\varphi)}{\delta}+\ln \delta
$$

and $b=b(\varphi)$ is a root of the quadratic equation

$$
0=\left((\lambda+\delta)^{2}-2 \varphi \xi^{2}\right) b^{2}-2\left(\lambda+\delta+2 \varphi \xi \rho_{e} \sigma_{e}\right) b+1-2 \varphi \sigma_{e}^{2}
$$

such that

$$
b(\varphi) \leq b(0)=\frac{1}{\lambda+\delta}
$$

Also for this last example, Proposition 12 implies a $O\left(\gamma^{2}\right)$-asymptotics for the equilibrium function $\widehat{g}$. This is stated by the next corollary.

Corollary 16 With the explicit model dynamics of Example 15 the function $\widehat{g}_{1}$ is the solution of the differential equation

$$
0=\mathbf{D}_{1}\left(\widehat{g}_{1}\right)+\mathbf{R}_{1}(X),
$$

where

$$
\begin{aligned}
& \mathbf{D}_{1}(g)=-\delta g-\left(\lambda(X-\bar{X})+\sqrt{2 \varphi} \xi \frac{\xi b(\varphi)+\rho_{e} \sigma_{e}}{\left(\sigma_{e}^{2}+2 \rho_{e} \sigma_{e} \xi b(\varphi)+(\xi b(\varphi))^{2}\right)^{\frac{1}{2}}} X\right) \frac{\partial g}{\partial X}+\frac{\xi^{2}}{2} X^{2} \frac{\partial^{2} g}{\partial^{2} X} \\
& \mathbf{R}_{1}(X)=\delta\left(\left(\frac{a(\varphi)+b(\varphi) X}{2}-1-\ln (\delta)\right)(a(\varphi)+b(\varphi) X)+\ln (\delta)\left(1+\frac{\ln (\delta)}{2}\right)\right) \\
& -\lambda(X-\bar{X}) b(\varphi)+\frac{\sigma_{e}^{2}+(\xi b(\varphi))^{2}+2 \xi \rho_{e} \sigma_{e} b(\varphi)}{2} X^{2}
\end{aligned}
$$

14 The drif term in the dynamics for $e$ in Example 15 is assumed quadratic in $X$ only in order to obtain a closed form solution for $\widehat{g}_{\log , \varphi}$. However, notice that for the kind of applications we have in mind $\sigma_{e} X$ is typically a small entity between 0 and 1 , so that in fact the linear drift part will dominate. 
and $a(\varphi)$ and $b(\varphi)$ are given in (63) and (64), respectively. Therefore $\widehat{g}_{1}$ is given by

$$
\widehat{g}_{1}(X)=\alpha(\varphi)+\beta(\varphi) X+\varepsilon(\varphi) X^{2}
$$

where

$$
\begin{aligned}
\varepsilon(\varphi)= & \frac{\sigma_{e}^{2}+2 \rho_{e} \sigma_{e} \xi b(\varphi)+\left(\delta+\xi^{2}\right) b(\varphi)^{2}}{2\left(\delta+2\left(\lambda+\sqrt{2 \varphi} \xi \frac{\xi b(\varphi)+\rho_{e} \sigma_{e}}{\left(\sigma_{e}^{2}+2 \rho_{e} \sigma_{e} \xi b(\varphi)+(\xi b(\varphi))^{2}\right)^{\frac{1}{2}}}\right)-\xi^{2}\right)} \\
\beta(\varphi)= & \frac{2 \lambda \bar{X}}{\delta+\lambda+\sqrt{2 \varphi} \xi \frac{\xi b(\varphi)+\rho_{e} \sigma_{e}}{\left(\sigma_{e}^{2}+2 \rho_{e} \sigma_{e} \xi b(\varphi)+(\xi b(\varphi))^{2}\right)^{\frac{1}{2}}}} \\
& +\frac{\delta\left(\left(\frac{1}{2} a(\varphi)-1-\ln \delta\right) b(\varphi)+\frac{1}{2} b(\varphi) a(\varphi)\right)-\lambda b(\varphi)}{\delta+\lambda+\sqrt{2 \varphi} \xi \frac{\xi b(\varphi)+\rho_{e} \sigma_{e}}{\left(\sigma_{e}^{2}+2 \rho_{e} \sigma_{e} \xi b(\varphi)+(\xi b(\varphi))^{2}\right)^{\frac{1}{2}}}} \\
\alpha(\varphi)= & \frac{\lambda \bar{X}}{\delta} \beta(\varphi)+\frac{\lambda \bar{X}}{\delta} b(\varphi)+\left(\left(\frac{1}{2} a(\varphi)-1-\ln \delta\right) a(\varphi)+(\ln \delta)\left(1+\frac{1}{2} \ln \delta\right)\right)
\end{aligned}
$$

Starting from Corollary 13, 14 and 16 we can now analyze analytically for some models the equilibrium impact of pessimism on asset prices, interest rates and risk premia by means of Propositions 8 and 10. Specifically, for the models in Example 5, 6 and 15, the obtained $O\left(\gamma^{2}\right)$ asymptotics for $\widehat{g}$ will allow us to investigate the indirect impact of pessimism on the equilibrium asset price dynamics and the implied worst case equity premia. On the other hand, for models where only $O(\gamma, \sqrt{\varphi})$ asymptotics for $\widehat{g}$ are available we will focus on the impact of pessimism on equilibrium interest rates and reference model equity premia.

\section{Some explicit settings of exogenous pessimism}

We work out a few explicit examples of a pessimistic exchange economy, starting from the general framework and the examples presented above, thereby providing the equilibrium formulae for $\widehat{\alpha}_{P}, \widehat{\sigma}_{P}$, $\widehat{\rho}$, and the equity risk premia $\widehat{\alpha}_{P}-\widehat{r}$ and $\left(\widehat{\alpha}_{P}-\widehat{r}\right)_{h *}$ in these economies. We achieve this by making explicit assumptions on the state process $Y$ and its confidence impact $f(X)$. Specifying the confidence process and its confidence impact gives specific shape to the agent's time-varying pessimism. In the final part of this section, we present two examples where this identification comes by linking agent's pessimism to some nonlinear function of the expected growth in dividends or the contemporaneous volatility in dividend growth.

\section{1 $O\left(\gamma^{3}\right)$-equilibrium asymptotics}

In this subsection we compute up to $O\left(\gamma^{3}\right)$-errors the equilibrium quantities implied by Proposition 8 and 10 for the Examples 5, 6, 15 in the previous sections. In particular, this enables us to investigate the impact of simple functional forms of exogenous pessimism on the equilibrium risky asset parameters $\widehat{\alpha}_{P}, \widehat{\sigma}_{P}, \widehat{\rho}$ and the worst case equity premium $\left(\widehat{\alpha}_{P}-\widehat{r}\right)_{h *}$. In section 5.2 below we will introduce some richer functional form for time varying pessimism. However, in this case only $O^{2}(\gamma, \sqrt{\varphi})$-equilibrium asymptotics can be obtained, so that the focus in that section will be on the impact on the reference model equity premium $\widehat{\alpha}_{P}-\widehat{r}$.

\subsubsection{Example 5}

By applying the previous results a $O\left(\gamma^{3}\right)$-asymptotics for the desired equilibrium variables in the setting of Example 5 is immediately obtained using Proposition 8. 
Corollary 17 The pessimistic exchange equilibrium implied by Example 5 yields conditional expectations of cumulative returns on equity, conditional variances and correlations given by

$$
\begin{aligned}
\widehat{\alpha}_{P}= & \alpha_{e}+\frac{\gamma}{1-\gamma}\left((b+\gamma(\beta(\varphi)+2 \varepsilon X))\left(-\lambda(X-\bar{X})+\xi \rho_{e} \sigma_{e}\right)\right) \\
& +\frac{\gamma}{1-\gamma} \frac{\xi^{2}}{2}\left(\frac{\gamma}{1-\gamma}((b+\gamma(\beta(\varphi)+2 \varepsilon X)))^{2}+2 \gamma \varepsilon\right) \\
& +\left(\frac{e^{\gamma\left(a(\varphi)+b X+\gamma\left(\alpha(\varphi)+\beta(\varphi) X+\varepsilon X^{2}\right)\right)}}{\delta}\right)^{\frac{1}{\gamma-1}}+O\left(\gamma^{3}\right), \\
\widehat{\sigma}_{P}^{2}= & \sigma_{e}^{2}+\frac{\gamma}{1-\gamma} 2 \rho_{e} \sigma_{e} \xi(b+\gamma(\beta(\varphi)+2 \varepsilon X))+\left(\frac{\gamma}{1-\gamma} \xi\right)^{2}((b+\gamma(\beta(\varphi)+2 \varepsilon X)))^{2}+O\left(\gamma^{3}\right), \\
\widehat{\rho}= & \frac{\sigma_{e} \rho_{e}+\frac{\gamma}{1-\gamma}(b+\gamma(\beta(\varphi)+2 \varepsilon X)) \xi}{\left(\sigma_{e}^{2}+\frac{\gamma}{1-\gamma} 2 \rho_{e} \sigma_{e} \xi(b+\gamma(\beta(\varphi)+2 \varepsilon X))+\left(\frac{\gamma}{1-\gamma} \xi\right)^{2}((b+\gamma(\beta(\varphi)+2 \varepsilon X)))^{2}\right)^{\frac{1}{2}}+O\left(\gamma^{3}\right),},
\end{aligned}
$$

respectively, where $a(\varphi), b, \alpha(\varphi), \beta(\varphi)$ and $\varepsilon$ are given in (22), (23), (52), (51) and (50), respectively.

Formulas (69)-(71) can be used to discuss analytically the impact of pessimism on the equilibrium price dynamics. For instance, from the conditional variance formula (70) we get the comparative statics

$$
\begin{aligned}
\left.\frac{\partial \widehat{\sigma}_{P}^{2}}{\partial \sqrt{\varphi}}\right|_{\varphi=0}= & \left.\frac{\partial \widehat{\sigma}_{P}^{2}}{\partial \beta} \frac{\partial \beta(\varphi)}{\partial \sqrt{\varphi}}\right|_{\varphi=0} \\
= & -\frac{2}{\lambda+\delta} \xi \frac{\xi b+\rho_{e} \sigma_{e}}{\left(\sigma_{e}^{2}+2 \rho_{e} \sigma_{e} \xi b+(\xi b)^{2}\right)^{\frac{1}{2}}} \varepsilon \\
& +\left.\frac{2 \gamma^{2}}{1-\gamma} \xi\left(\rho_{e} \sigma_{e}+\frac{\gamma}{(1-\gamma)} \xi(b+\gamma(\beta(0)+2 \varepsilon X))\right) \frac{\delta b}{\lambda+\delta} \cdot \frac{\partial a(\varphi)}{\partial \sqrt{\varphi}}\right|_{\varphi=0}+O\left(\gamma^{3}\right)
\end{aligned}
$$

using (51). Furthermore, by (22),

$$
\left.\frac{\partial a(\varphi)}{\partial \sqrt{\varphi}}\right|_{\varphi=0}=-\frac{\sqrt{2}}{\delta}\left(\sigma_{e}^{2}+\frac{2 \rho_{e} \sigma_{e} \xi}{\lambda+\delta}+\left(\frac{\xi}{\lambda+\delta}\right)^{2}\right)^{\frac{1}{2}}<0
$$

Therefore, the marginal impact of pessimism on the equilibrium volatility of equity can be characterized in terms of the model parameters, the state $X$ and the risk aversion parameter. Similar, but more involved, comparative statics can be developed also for $\widehat{\alpha}_{P}, \widehat{\rho}$, and the reference model and worst case equity premia of Proposition 10. Due to the complexity of the implied expressions, we illustrate the obtained quantities graphically based on some plausible structural parameter values.

We calibrate our models using the unconditional moments of US historical consumption growth. In choosing model parameters we use historical per capita consumption over the 1889 - 1994 period. Consumption data are real with annual frequency. We choose the drift and diffusion of the dividend process $\left(e_{t}\right)$ to match the corresponding values of per capita aggregate consumption. This puts restrictions on the parameters governing the state process $X$ as well. We take the estimates of the unconditional moments of the consumption growth from Table 8.1, pg. 308, of Campbell, Lo, 
and MacKinlay (1997). They define consumption growth as the change in log real consumption of nondurables and services. Given the annual frequency, the theoretical counterpart of such observable is $\int_{0}^{1} d \ln e$.

\begin{tabular}{|c||c|c|}
\hline Variable & Mean & Standard deviation \\
\hline \hline Consumption growth & 0.0172 & 0.0328 \\
\hline
\end{tabular}

Because consumption of nondurables and services is a smooth series, equity looks like a small bet in this exchange economy. The long-run standard deviation of its dividends is only about $3.3 \%$.

A reasonable magnitude for the pessimism parameter $\varphi$ can be determined in the context of statistical model discrimination, as in Anderson, Hansen, and Sargent (2000). To highlight this point assume that the pessimistic agent is faced with two relevant models of the economy: the reference model and the worst case model. Distorted models implied by large $\varphi$ 's are statistically easy to tell apart from the reference model when the investor has a large data set of past observations. With a data set of size N, Anderson, Hansen, and Sargent (2000) show that the upper bound on the probability of failing to distinguishing between the reference model and the worst case model is:

$$
\text { probability bound }=\frac{1}{2} \exp \left(-N \frac{\varphi}{4}\right) \text {. }
$$

The annual frequency of the consumption data over the considered century-long period implies $N=100$. However, the agent may well want to use quarterly century-long data in order to do better inference. Thus, a prudent choice of the level of economy-wide pessimism originates from $N=400$ to yield a probability bound of 0.11 . The corresponding level of $\varphi$ is 0.015 . To summarize,

$$
0.11=\frac{1}{2} \exp \left(-400 \cdot \frac{0.015}{4}\right)
$$

We generate a century-long sample of quarterly data from the processes that drive the economy in Example 5. We take the following liberty in tuning the parameters of these processes. We force the unconditional mean and standard deviation of $\int_{0}^{1} d \ln e$ to equal the unconditional mean and standard deviation of the annualized quarterly changes in the log-dividends, $\frac{1}{\Delta t} \Delta \ln e$ with $\Delta t=3 / 12$. The calibration of the state and dividend processes $\left(X_{t}\right)$ and $\left(e_{t}\right)$ gives

$$
\begin{aligned}
d X & =-0.5\left(X-\left(0.0172+0.5 \cdot 0.0328^{2}\right)\right) d t+0.0328 \cdot d Z^{X} \\
d \ln e & =\left(X-0.5 \cdot 0.0328^{2}\right) d t+0.0328 \cdot\left[0.5 d Z^{X}+\sqrt{1-0.5^{2}} d Z^{e}\right] .
\end{aligned}
$$

The adjustment-speed parameter $\lambda$ has been set to 0.5 so that the long-run volatility of forecast consumption growth $(\xi / \sqrt{2 \lambda})$ equals 0.0328 . The correlation coefficient $\rho_{e}$ has been set to 0.5 to match a century-long correlation between S\&P 500 equity return and consumption growth of about 0.5. The time preference rate $\delta$ is set equal to 0.053 like in the calibration exercise by Kogan and Chan (2001).

The results of our calibration exercise are summarized in Figure 1 and 2. Figure 1 focuses on the implied equity premia and worst case equity premia.

\section{Insert Figure 1 about here}

In the bottom left panel of Figure 1 it is shown that the given model dynamics imply a time invariant equity premium, both for economies with and without pessimism. The premium implied by the presence of pessimism is more than five times the one implied by a comparable non-pessimistic economy, clearly illustrating the amplitude of FORA effects on excess expected returns. The unconditional equity premium ${ }^{15}$ with $(\gamma=-0.10, \varphi=0.015)$ is 45 basis points, with a spread of 34 basis points

\footnotetext{
${ }^{15}$ We simulate 1000 paths of conditional equity premia for 400 quarters and we measure the unconditional premium as the cross-sectional mean of the 1000 conditional premia at the 400th quarter.
} 
on the case $(\gamma=-0.10, \varphi=0)$. The top left panel of Figure 1 illustrates the trade-off between risk aversion and pessimism in determining equity premia. Specifically, we see that in this model higher risk aversions require a higher degree of pessimism to maintain a given level of equity premium. This pattern derives from two opposite effects. First, higher risk and model uncertainty aversions imply directly a positive first order effect on equity premia, deriving from a pure substitution of risky with riskless assets. Second, higher risk aversions imply a lower desire to substitute intertemporally, and thereby higher interest rates. For the present model, this second effect is compensated only via a higher degree of pessimism in the economy. Moreover, the larger the risk aversion the larger this second effect seems to be.

Finally, the top and bottom right panels of Figure 1 illustrate the impacts on worst case equity premia. Basically, we observe that in this model the presence of pessimism implies lower worst case premia. However, the entity of this effect is virtually negligible. Indeed, the worst case premium level curves in the top right panel of Figure 1 imply an almost constant surface and are almost orthogonal to the risk aversion axis, implying that they are not significantly influenced by pessimism.

Figure 2 summarizes the impact of pessimism on equity dynamics and the equilibrium asymptotics for the function $\widehat{g}$.

\section{Insert Figure 2 about here}

As expected, $\widehat{g}$ is asymptotically reduced by the presence of pessism (cf. the bottom right panel in Figure 2), as a consequence of the max-min optimization behavior of a pessimistic agent. Further, expected equity returns, volatilities and correlations are readily unaffected by the presence of pessimism. Therefore, in this model the time invariant pessimistic equity premia in Figure 1 are determined by interest rates that basically offset a corresponding change in expected returns.

\subsubsection{Example 6}

The $O\left(\gamma^{3}\right)$-asymptotics implied for the equilibrium equity dynamics in Example 6 are given in the next corollary.

Corollary 18 The pessimistic exchange equilibrium implied by Example 6 yields conditional expectations of cumulative returns on equity, conditional variances and correlations given by

$$
\begin{aligned}
\widehat{\alpha}_{P}= & \alpha_{e}+\frac{\gamma}{1-\gamma}\left((b(\varphi)+\gamma(\beta(\varphi)+2 \varepsilon(\varphi) X))\left(-\lambda(X-\bar{X})+\xi \rho_{e} \sigma_{e} X\right)\right) \\
& +\frac{\gamma}{1-\gamma} \frac{\xi^{2}}{2} X\left(\frac{\gamma}{1-\gamma}((b(\varphi)+\gamma(\beta(\varphi)+2 \varepsilon(\varphi) X)))^{2}+2 \varepsilon \gamma(\varphi)\right) \\
& +\left(\frac{\left.e^{\gamma\left(a(\varphi)+b(\varphi) X+\gamma\left(\alpha(\varphi)+\beta(\varphi) X+\varepsilon(\varphi) X^{2}\right)\right)}\right)^{\frac{1}{\gamma-1}}+O\left(\gamma^{3}\right)}{\delta},\right. \\
\widehat{\sigma}_{P}^{2}= & \left(\sigma_{e}^{2}+\frac{\gamma}{1-\gamma} 2 \rho_{e} \sigma_{e} \xi(b(\varphi)+\gamma(\beta(\varphi)+2 \varepsilon(\varphi) X))\right) X \\
& +\left(\frac{\gamma}{1-\gamma} \xi\right)^{2}(b(\varphi)+\gamma(\beta(\varphi)+2 \varepsilon(\varphi) X))^{2} X+O\left(\gamma^{3}\right), \\
\widehat{\rho}= & \frac{\sigma_{e} \rho_{e}+\frac{\gamma}{1-\gamma}(b(\varphi)+\gamma(\beta(\varphi)+2 \varepsilon(\varphi) X)) \xi}{\left(\sigma_{e}^{2}+\frac{\gamma}{1-\gamma} 2 \rho_{e} \sigma_{e} \xi(b(\varphi)+\gamma(\beta(\varphi)+2 \varepsilon(\varphi) X))+\left(\frac{\gamma}{1-\gamma} \xi\right)^{2}((b(\varphi)+\gamma(\beta(\varphi)+2 \varepsilon(\varphi) X)))^{2}\right)^{\frac{1}{2}}} \\
& +O\left(\gamma^{3}\right),
\end{aligned}
$$

respectively, where $a(\varphi), b(\varphi), \alpha(\varphi), \beta(\varphi)$ and $\varepsilon(\varphi)$ are given in (30), (31), (54), (55) and (56), respectively. 
We illustrate again the properties of the implied pessimistics equity dynamics and equity premia with some graphs. For this setting, the calibration of the model dynamics gives

$$
\begin{aligned}
d X & =-3\left(X-(0.0328)^{2}\right) d t+\xi \sqrt{X} d Z^{X} \\
d \ln e & =\left(0.0177-0.5 \cdot(1.00)^{2} X\right) d t+(1.00) \sqrt{X}\left[(-0.25) d Z^{X}+\sqrt{1-(-0.25)^{2}} d Z^{e}\right] .
\end{aligned}
$$

The long run mean of $d \ln e$ has been set equal to the estimated long run mean of consumption growth,

$$
0.0177-0.5(1.00)^{2}(0.0328)^{2}=0.0172
$$

The adjustment-speed parameter $\lambda$ is 3 . The coefficient $\xi^{2}$ is a fraction of $2 \lambda \bar{X}$,

$$
\xi^{2}=0.75(2 \lambda \bar{X}) \leq 2 \cdot 3 \cdot(0.0328)^{2} .
$$

This preserves positivity and ergodicity of the conditional variance of the consumption growth. The correlation coefficient $\rho_{e}$ has been set to -0.25 to match the intuition that jitters in the economy (high conditional variance of consumption growth) are associated with bad economy-wide news.

The implied equilibrium quantities are ilustrated in Figures 3 and 4 . From Figure 3 we can observe partly similar patterns as the ones showed in the previous example.

\section{Insert Figure 3 about here}

First, we see from the top and bottom left panels of Figure 3 that pessimism induces again clearly higher equity premia for any given level of the state $X$ in the economy. Therefore, FORA again contributes substantially to higher expected excess returns. The unconditional equity premium with $(\gamma=-0.10, \varphi=0.015)$ is 68 basis points, with a spread of 56 basis points on the case $(\gamma=-0.10, \varphi=0)$. Further, since now volatility and pessimism are state dependent, the volatility of equity premia is larger in the presence of pessimism. This is a combined effect of FORA and state dependent pessimism. As in the previous examples the top and bottom right panels in Figure 3 show that worst case premia are altered only in a negligible way by the presence of pessimism. As in the previous case they are fairly small because they are determined essentially only by standard risk aversion. Figure 4 plots the implied equity returns moments and asymptotic approximations for the equilibrium function $\widehat{g}$.

\section{Insert Figure 4 about here}

As expected, the asymptotic expression for $\widehat{g}$ is lower in the presence than in the absence of pessimism while equilibrium expected returns, volatilities and correlations are only negligibly influenced.

\subsubsection{Example 15}

The $O\left(\gamma^{3}\right)$-asymptotics implied for the equilibrium equity dynamics in Example 15 are as follows.

Corollary 19 The pessimistic exchange equilibrium implied by Example 15 yields conditional expec- 
tations of cumulative returns on equity, conditional variances and correlations given by

$$
\begin{aligned}
\widehat{\alpha}_{P}= & X+\frac{1}{2} \sigma_{e}^{2} X^{2}+\frac{\gamma}{1-\gamma}\left((b(\varphi)+\gamma(\beta(\varphi)+2 \varepsilon(\varphi) X))\left(-\lambda(X-\bar{X})+\xi \rho_{e} \sigma_{e} X^{2}\right)\right) \\
& +\frac{\gamma}{1-\gamma} \frac{\xi^{2}}{2} X^{2}\left(\frac{\gamma}{1-\gamma}((b(\varphi)+\gamma(\beta(\varphi)+2 \varepsilon(\varphi) X)))^{2}+\gamma 2 \varepsilon(\varphi)\right) \\
& +\left(\frac{e^{\gamma\left(a(\varphi)+b(\varphi) X+\gamma\left(\alpha(\varphi)+\beta(\varphi) X+\varepsilon(\varphi) X^{2}\right)\right)}}{\delta}\right)^{\frac{1}{\gamma-1}}+O\left(\gamma^{3}\right) \\
\widehat{\sigma}_{P}^{2}= & \left(\sigma_{e}^{2}+\frac{\gamma}{1-\gamma} 2 \rho_{e} \sigma_{e} \xi(b(\varphi)+\gamma(\beta(\varphi)+2 \varepsilon(\varphi) X))\right) X^{2} \\
& +\left(\frac{\gamma}{1-\gamma} \xi\right)^{2}((b(\varphi)+\gamma(\beta(\varphi)+2 \varepsilon(\varphi) X)))^{2} X^{2}+O\left(\gamma^{3}\right), \\
\widehat{\rho}= & \frac{\sigma_{e} \rho_{e}+\frac{\gamma}{1-\gamma}(b(\varphi)+\gamma(\beta(\varphi)+2 \varepsilon(\varphi) X)) \xi}{\left(\sigma_{e}^{2}+\frac{\gamma}{1-\gamma} 2 \rho_{e} \sigma_{e} \xi(b(\varphi)+\gamma(\beta(\varphi)+2 \varepsilon(\varphi) X))+\left(\frac{\gamma}{1-\gamma} \xi\right)^{2}((b(\varphi)+\gamma(\beta(\varphi)+2 \varepsilon(\varphi) X)))^{2}\right)^{\frac{1}{2}}} \\
& +O\left(\gamma^{3}\right),
\end{aligned}
$$

respectively, where $a(\varphi), b(\varphi), \alpha(\varphi), \beta(\varphi)$ and $\varepsilon(\varphi)$ are given in (63), (64), (68), (67) and (66), respectively.

As for the previous examples, we illustrate the properties of the implied pessimistic equity dynamics and equity premia with some graphs. The calibration of the model dynamics now gives

$$
\begin{aligned}
d X & =-3(X-0.0172) d t+\xi X d Z^{X} \\
d \ln e & =X d t+(0.0328 / 0.0172) X\left[0.1 d Z^{X}+\sqrt{1-0.1^{2}} d Z^{e}\right] .
\end{aligned}
$$

The long run mean of $\left(X_{t}\right)$ is 0.0172 . Moreover, in this model the state process again also as the conditional volatility of dividend growth. To accomodate this we have set $\sigma_{e} \bar{X}=0.0328$. The adjustment-speed parameter $\lambda$ is 3 . The coefficient $\xi^{2}$ is a fraction of $2 \lambda \bar{X}$,

$$
\xi^{2}=0.75(2 \lambda \bar{X}) \leq 2 \cdot 3 \cdot(0.0328)^{2}
$$

This calibration has been performed partly in a similar vain as for the previous CIR case in order to make the predictable mean-reverting component of $\left(X_{t}\right)$ dominate on the random walk of the news. The correlation coefficient $\rho_{e}$ is 0.1 to match the intuition that a high conditional expected consumption growth is associated with good economy-wide news. Figures 5 and 6 summarize graphically the results of our calibration.

\section{Insert Figure 5 about here}

Figure 5 mimics the impact of pessimism on equity premia and worst case equity premia. Again, we find a sizable FORA effect on equity premia (cf. the top and bottom left panel of Figure 5), which are about four times higher in the presence of pessimism. The unconditional equity premium with $(\gamma=-0.10, \varphi=0.015)$ is 68 basis points, with a spread of 57 basis points on the case $(\gamma=-0.10, \varphi=0)$. By constrast, worst case premia are again virtually unaffected by pessimism, with worst case equity premium level curves that are almost orthogonal to the risk aversion axis. At variance with Figure 1, the equity premium level curves in the top left panel of Figure 5 are now slightly positively sloped, indicating that the direct impact of pessimism on equity premia dominates over the effect of a lower intertemporal substitution rate associated with higher risk aversion parameters. 
This suggests that in this models the implied equity premia can be further increased by highering the risk aversion parameter without the need of introducing irrealistically high amounts of pessimism. Further, in this model equity premia are state dependent, with a variability that is higher than in the absence of pessimism (cf. the bottom left panel of Figure 1).

Figure 6 summarizes the structure of the implied equity returns moments and the symptotic approximations for the equilibrium function $\widehat{g}$.

\section{Insert Figure 6 about here}

The message is very similar to the one obtained for the previous models. Indeed, as expected the asymptotic equilibrium funtion $\widehat{g}$ is lower in the presence of pessimism (cf. the bottom right panel of Figure 6), while the moments of the equity dynamics are influenced in a virtually negligible way.

\section{$5.2 O^{2}(\gamma, \sqrt{\varphi})$ - equilibrium asymptotics}

We conclude our analysis of pessimistic equilibria with two examples where the maximal distrust function $f(X)$ reflects forward looking expectations of future dividends and mean volatilities relatively to some current benchmark level. By contrast with the former analysis, specifying a richer structure $f(X)$ for state dependent pessimism allows only for pessimistic equilibrium asymptotics of the order $O^{2}(\gamma, \sqrt{\varphi})$.

\subsubsection{Example 5 with state dependent pessimism}

We further illustrate the equilibrium impact of a concern for pessimism in cases where the opportunity set process $X$ only affects the short-run forecast for the dividend growth $E_{t}\left(\frac{d e}{e}\right)$. Agent's reference belief on forecast dividend growth, actual dividend growth, and equilibrium cumulative returns on equity is given by the dynamics of Example 5 .

We assume that the function $f$ describing the confidence impact of $X$ is the reference model discounted conditional expectation of the negative cumulative dividend growth over some time horizon $h>0$, that is

$$
f(X)=E\left(\frac{e_{0}}{e_{h}} \mid X_{0}=X\right) .
$$

Notice that in equilibrium $e$ is the aggregate consumption in this economy. Therefore, in equilibrium $f(X)$ will generate a higher misconfidence in the reference belief at time $t$, precisely when the expected consumption growth over the time period $(t, t+h]$ is low, and vice versa. Standard calculations using the given model dynamics imply ${ }^{16}$

$$
f(X)=\exp (-A(h)-B(h) X),
$$

where

$$
B(h)=\frac{1-\exp (-\lambda h)}{\lambda} \quad, \quad A(h)=\left(\frac{\xi^{2}}{2 \lambda^{2}}-\bar{X}\right)(B(h)-h)+\frac{\xi^{2}}{4 \lambda} B(h)^{2}
$$

Notice, that in this setting $f(X)$ discounts states $X$ with higher instantaneous expected dividend growth into a higher confidence for the reference belief, that is a lower maximal distrust $f(X)$ in the allowed drift misspecifications. The way by which the maximal distrust is determined depends on the the prediction horizon $h$. The function $\widehat{g}_{\log , 0}$ in this setting is, by the results in Example 5 for $\varphi=0$ :

$$
\widehat{g}_{0 \log }(X)=a(0)+b X+\log \delta,
$$

where $a(0)$ and $b$ are given in (22) and (23), respectively. Therefore, we can readily apply the findings of the previous sections to provide the desired $O^{2}(\gamma, \sqrt{\varphi})$-equilibrium asymptotics.

\footnotetext{
${ }^{16}$ For completeness, a proof is given in the Appedix.
} 
Proposition 20 Given the primitives of Example 5 and the pessimistic parameterization (79), the pessimistic exchange equilibrium implies conditional expectations of cumulative returns on equity given by

$$
\widehat{\alpha}_{P} \doteq(1-\gamma) X+\delta+\gamma \rho_{e} \sigma_{e} \xi b+\gamma \frac{\sigma_{e}^{2}}{2}
$$

conditional variances given by

$$
\widehat{\sigma}_{P}^{2} \stackrel{\circ}{=} \sigma_{e}\left(1-\gamma \frac{2 \rho_{e}}{\sigma_{e}} \xi b\right),
$$

conditional correlations with the the state variable $X$ given by

$$
\widehat{\rho} \doteq \frac{\rho_{e}-\gamma \frac{\xi b}{\sigma_{e}}}{\sqrt{1-\gamma \frac{2 \rho_{e}}{\sigma_{e}} \xi b}}
$$

and conditional reference model equity premia given by

$$
\begin{aligned}
\widehat{\alpha}_{P}-\widehat{r} \stackrel{\circ}{=} & \sigma_{e}^{2}\left(1-\gamma \frac{2 \rho_{e}}{\sigma_{e}} \xi b\right) \\
& -\left(\gamma-\sqrt{\frac{2 \varphi}{\sigma_{e}^{2}+(\xi b)^{2}+2 \rho_{e} \sigma_{e} \xi b}} \exp (-A(h)-B(h) X)\right)\left(\sigma_{e}^{2}+\rho_{e} \sigma_{e} \xi b\right)
\end{aligned}
$$

In this model expected returns on equity are (to first order) linear functions of instantaneous expected endowment returns $X$ and behave procyclically with $X$, while equilibrium equity volatilities are constant. Further, in the absence of pessimism $(\varphi=0)$ equity risk premia are constant, implying procyclical equilibrium interest rates. When introducing pessimism $(\varphi>0)$ into the analysis equity premia and sharpe ratios behave countercyclically with $X$ because countercyclical effective risk aversion causes a countercyclical behavior in the maximal drift distrust exp $\left(-A_{1}(h)-B_{1}(h) X\right)$. In that case, the procyclical behavior of equilibrium interest rates does not fully compensate the procyclical movements in equilibrium expected returns on equity.

Figure 7 summarizes graphically the impact of pessimism on equity premia for this model, highlightening again the significance of FORA effects already for moderate amounts of parameterized pessimism.

\section{Insert Figure 7 about here}

Specifically, by contrast with a constant parameterization of pessimism equity premia are now cyclical as functions of the pessimistic maximal distrust $f(X)$.

Finally, remark that the functional form (79) for the maximal distrust in the worst case drift under model misspecification is readily a free parameter for the model builder. For instance, one coud adopt a smoothed, slowly-varying version of the expected cumulative dividend growth (79) by defining $f(X)$ as an expected geometric average of future dividend growths (or equivalently future equilibrium consumption growths) given by

$$
f(X)=E\left(\frac{e_{0}}{\exp \left(\bar{e}_{h}\right)} \mid X_{0}=X\right),
$$

where

$$
\bar{e}_{h}=\log \left(e_{0}\right)+\vartheta \int_{0}^{h} \exp (-\vartheta t) \log \left(e_{t}\right) d t \quad, \quad \vartheta>0 .
$$

The calculations for such a specification are identical to the ones for the specification (79) since under the given assumptions $\left(\bar{e}_{t}\right)$ is again a mean reverting Ornstein Uhlembeck process. 


\subsubsection{Example 6 with a further parameterization of pessimism}

The last example illustates further the equilibrium implications of time varying pessimism for cases where it depends nonlinearly on the conditional variance of dividend growth $\operatorname{Var}_{t}\left(\frac{d e}{e}\right)$. Agent's reference belief on the volatility of dividend growth innovations, actual dividend growth, and equilibrium cumulative returns on equity are as in Example 6. A negative $\rho_{e}$ captures the stylized fact that in capital markets bad news imply higher volatility. Further, in this model forecast dividend growth coincides with its long-run average $\alpha_{e}>0$.

We specify as an indicator of model confidence the ratio between the current variance of dividend growth and the mean expected variance of dividend growth over some time horizon $h>0$ :

$$
f(X)=\left(\frac{h \cdot \operatorname{Var}_{0}\left(\frac{d e}{e}\right)}{E_{0}\left(\int_{0}^{h} \operatorname{Var}_{t}\left(\frac{d e}{e}\right) d t \mid X_{0}=X\right)}\right) .
$$

This parameterization increases agent's distrust in the reference model precisely when current variances of the dividend growth are higher relatively to expected future mean variances. Thus, risk premia deflate with a lower volatility of aggregate consumption growth.

Standard computations using the explicit model setting in Example 6 then give

$$
E\left(\int_{0}^{h} \operatorname{Var}_{t}\left(\frac{d e}{e}\right) d t \mid X_{0}=X\right)=\bar{X} h+(X-\bar{X}) \frac{1-\exp (-\lambda h)}{\lambda},
$$

and the following explicit expression for (82)

$$
f(X)=\frac{X}{\bar{X}+\left(\frac{1-\exp (-\lambda h)}{\lambda h}\right)(X-\bar{X})} .
$$

Finally, the function $\widehat{g}_{0} \log$ in this model is obtained by the results in Example 6 for $\varphi=0$ :

$$
\widehat{g}_{\log , 0}(X)=a(0)+b(0) X+\ln (\delta) \quad,
$$

where $a(0)$ and $b(0)$ are given in (30) and (31), respectively. The implied equilibrium asymptotics are given in the next last proposition.

Proposition 21 Given the primitives of Example 6 and the pessimistic parameterization (82), the pessimistic exchange equilibrium implies conditional expectations of cumulative returns on equity given by

$$
\widehat{\alpha}_{P} \stackrel{\circ}{=}(1-\gamma) \alpha_{e}+\delta+\gamma\left(\rho_{e} \sigma_{e} \xi b_{2}+\frac{\sigma_{e}^{2}}{2}\right) X
$$

conditional volatilities given by

$$
\widehat{\sigma}_{P} \stackrel{\circ}{=} \sigma_{e} \sqrt{X\left(1-\gamma \frac{2 \rho_{e}}{\sigma_{e}} \xi b_{2}\right)},
$$

and a conditional correlation with the the state variable $X$ is given by

$$
\widehat{\rho} \doteq \frac{\rho_{e}-\gamma \frac{\xi b_{2}}{\sigma_{e}}}{\sqrt{1-\gamma \frac{2 \rho_{e}}{\sigma_{e}} \xi b_{2}}} .
$$


Finally, the conditional reference model equity premium is

$$
\begin{aligned}
\widehat{\alpha}_{P}-\widehat{r} \doteq & \sigma_{e}^{2}\left(1+\gamma \frac{2 \rho_{e}}{\sigma_{e}} \xi b_{2}\right) \\
& -\left(\sigma_{e}^{2}+\rho_{e} \sigma_{e} \xi b_{2}\right)\left(\gamma X-\sqrt{\frac{2 \varphi X}{\left(\sigma_{e}^{2}+\left(\xi b_{2}\right)^{2}+2 \rho_{e} \sigma_{e} \xi b_{2}\right)}} \cdot \frac{X}{\bar{X}+\left(\frac{1-\exp (-\lambda h)}{\lambda h}\right)(X-\bar{X})}\right) .
\end{aligned}
$$

In this model expected returns are linear functions of conditional variances of endowment returns. Further, assuming $\rho_{e} \sigma_{e} \xi b_{2}>0$ (a typical parameter condition for these models) and for $\gamma<0$ expected returns behave countercyclically with $X$. Moreover, in the absence of pessimism $(\varphi=0)$ equity premia are a linear function of conditional variances of endowment returns. For $\rho_{e} \sigma_{e} \xi b_{2}>0$ and $\gamma<0$ this function is monotonically increasing, so that equity premia increase with instantaneous dividend growth variances $X$.

On the other hand, when pessimism is present we observe that equity premia are linearly related also to the volatility of dividend growth, multiplied by the given indicator of pessimism in the economy. For instance, when $X=\bar{X}$, the part of equity premium induced by a concern for model misspecification is

$$
\left(\sigma_{e}^{2}+\rho_{e} \sigma_{e} \xi b_{2}\right) \cdot \sqrt{\frac{2 \varphi \bar{X}}{\left(\sigma_{e}^{2}+\left(\xi b_{2}\right)^{2}+2 \rho_{e} \sigma_{e} \xi b_{2}\right)}} .
$$

In this case, the pessimistic part of the conditional equity premium is proportional to the volatility factor $\sqrt{X}$, showing a FORA effect of a pessimistic concern for misspecification on equity premia. Further, if $X>\bar{X}(X<\bar{X})$ this basic FORA effect is amplified (reduced) by the multiplicative factor

$$
\frac{X}{\bar{X}+\left(\frac{1-\exp (-\lambda h)}{\lambda h}\right)(X-\bar{X})},
$$

that reflects the further impact of a higher (lower) conditional model uncertainty associated to a higher (lower) current variance level $\operatorname{Var}_{t}\left(\frac{d e}{e}\right)$ relatively to the mean level expected over some finite horizon in the future.

Figure 8 summarizes graphically the impact of pessimism on equity premia for this model, quantifying again the significance of FORA effects already for moderate amounts of parameterized pessimism in the presence of stochastic volatility.

\section{Insert Figure 8 about here}

Specifically, by contrast with a constant parameterization of pessimism equity premia and their volatilities are now cyclical as functions of the pessimistic maximal distrust $f(X)$.

\section{Conclusions}

We presented a flexible analytical framework in which the equilibrium impact of state dependent pessimism in continuous time intertemporal asset pricing can be described and analyzed under fairly general assumptions, including state dependent opportunity sets and existence of intermediate consumption. We circumvented the quasi-intractability of the implied optimization problems by adopting a perturbative approach to compute finite order approximations for our exchange equilibrium asset prices. We have shown that pessimism does not affect in a quantitatively important way the stochastic process of the equilibrium equity price and the implied worst case equity premia. In our setting 
pessimism yields an asset substitution between equity and bonds that produces lower equilibrium interest rates and significantly higher equity premia via FORA effects. This dichotomy of the effects on equity premia and equity dynamics suggests that a more general pessimistic setting with for instance some agents heterogeneity or a utility function of intermediate consumption different from the standard CRRA form used in this paper could help in jointly fitting a high equity premium, a low interest rate and some given structural parameters of equilibrium equity dynamics.

\section{Appendix}

In this appendix we provide the proofs to the propositions in the paper.

Proof of Proposition 1. Minimizing $u(c W)-\delta J+\frac{1}{d t} E_{t}^{h}(d J)$ with respect to the contaminating vector $h$ under the binding constraint on $h$ 's size yields

$$
h^{*}=-\frac{1}{\lambda} \Lambda^{\prime} J_{Y}, \quad \frac{1}{2} h^{* \prime} h^{*}=\varphi f^{2}(X),
$$

where $\lambda$ is the Lagrangean multiplier. The binding constraint implies that

$$
\lambda=\frac{\left(J_{Y}^{\prime} \Lambda \Lambda^{\prime} J_{Y}\right)^{\frac{1}{2}}}{\sqrt{2 \varphi}|f(X)|}
$$

and this completes the proof.

Proof of Proposition 2. The single agent HJB equation implied by the worst case scenario in Proposition 1 is

$$
\begin{aligned}
0= & \sup _{c, w}\left\{\frac{(c W)^{\gamma}-1}{\gamma}-\delta J+\left(w W\left(\alpha_{P}-r\right)+(r W-c W)\right) J_{W}+\frac{1}{2} w^{2} W^{2} \sigma_{P}^{2} J_{W W}\right. \\
& \left.+\zeta J_{X}+\frac{1}{2} \xi^{2} J_{X X}+w W \rho \sigma_{P} \xi J_{X W}-\sqrt{2 \varphi}|f(X)|\left[w^{2} W^{2} \sigma_{P}^{2} J_{W}^{2}+\xi^{2} J_{X}^{2}+2 w W \rho \sigma_{P} \xi J_{W} J_{X}\right]^{\frac{1}{2}}\right\} .
\end{aligned}
$$

Using the functional form (4) for a solution, the implied optimal policies are given by

$$
\begin{aligned}
c^{*} & =\frac{\left(J_{W}\right)^{\frac{1}{\gamma-1}}}{W}=\left(\frac{e^{\gamma g}}{\delta}\right)^{\frac{1}{\gamma-1}}, \\
w^{*} & =-\frac{1}{1-\sqrt{\frac{2 \varphi}{\Gamma\left(w^{*}\right)}}|f(X)| \frac{J_{W}^{2}}{J_{W W}}} \cdot \frac{J_{W}}{W J_{W W}}\left(\frac{\alpha_{P}-r}{\sigma_{P}^{2}}+\frac{J_{W X}}{W J_{W W}} \frac{\rho \xi}{\sigma_{P}}-\sqrt{\frac{2 \eta}{\Gamma\left(w^{*}\right)}} J_{X} \frac{\rho \xi}{\sigma_{P}}\right) \\
& =\frac{1}{1-\sqrt{\frac{2 \varphi}{\Gamma\left(w^{*}\right)}}|f(X)| \frac{J_{W}^{2}}{J_{W W}}} \cdot \frac{1}{1-\gamma}\left(\frac{\alpha_{P}-r}{\sigma_{P}^{2}}+\gamma \frac{\partial g}{\partial X} \cdot \frac{\rho \xi}{\sigma_{P}}-\sqrt{\frac{2 \eta}{\Gamma\left(w^{*}\right)}} J_{X} \frac{\rho \xi}{\sigma_{P}}\right),
\end{aligned}
$$

where

$$
\begin{aligned}
\Gamma(w) & =W^{2} J_{W}^{2}\left(\sigma_{P}^{2} w^{2}+\frac{\xi^{2} J_{X}^{2}}{W^{2} J_{W}^{2}}+2 w \rho \sigma_{P} \xi \frac{J_{X}}{W J_{W}}\right) \\
& =W^{2} J_{W}^{2}\left(\sigma_{P}^{2} w^{2}+\xi^{2}\left(\frac{\partial g}{\partial X}\right)^{2}+2 w \rho \sigma_{P} \xi \frac{\partial g}{\partial X}\right) \\
& =W^{2} J_{W}^{2} G(w)
\end{aligned}
$$


with $G$ defined accordingly. Therefore, the optimal investment policy reads more compactly

$$
w^{*}=\frac{1}{1-\left(\gamma-\sqrt{\frac{2 \varphi}{G\left(w^{*}\right)}}|f(X)|\right)} \cdot\left(\frac{\alpha_{P}-r}{\sigma_{P}^{2}}+\left(\gamma-\sqrt{\frac{2 \varphi}{G\left(w^{*}\right)}}|f(X)|\right) \frac{\partial g}{\partial X} \frac{\rho \xi}{\sigma_{P}}\right) .
$$

Using the expansions

$$
\begin{aligned}
w^{*}(X) & =\frac{\alpha_{P}-r}{\sigma_{P}^{2}}+\gamma w_{1}(X)+\sqrt{2 \varphi} w_{2}(X)+O^{2}(\gamma, \sqrt{\varphi}), \\
g(X) & =g_{0}(X)+\gamma g_{1}(X)+\sqrt{2 \varphi} g_{2}(X)+O^{2}(\gamma, \sqrt{\varphi}),
\end{aligned}
$$

the result follows by expanding the above exact optimal policies to first order in $(\gamma, \sqrt{\varphi})$.

Proof of Theorem 4. The relevant single agent HJB equation is

$$
\begin{aligned}
0= & \sup _{c, w}\left\{\frac{(c W)^{\gamma}-1}{\gamma}-\delta J+\left(w W\left(\alpha_{P}-r\right)+(r W-c W)\right) J_{W}+\frac{1}{2} w^{2} W^{2} \sigma_{P}^{2} J_{W W}\right. \\
& \left.+\zeta J_{X}+\frac{1}{2} \xi^{2} J_{X X}+w W \rho \sigma_{P} \xi J_{X W}-\sqrt{2 \varphi}|f(X)|\left[w^{2} W^{2} \sigma_{P}^{2} J_{W}^{2}+\xi^{2} J_{X}^{2}+2 w W \rho \sigma_{P} \xi J_{W} J_{X}\right]^{\frac{1}{2}}\right\},
\end{aligned}
$$

with optimal policies given by

$$
\begin{aligned}
c^{*} & =\frac{\left(J_{W}\right)^{\frac{1}{\gamma-1}}}{W}=\left(\frac{e^{\gamma g}}{\delta}\right)^{\frac{1}{\gamma-1}}, \\
w^{*} & =\frac{1}{1-\left(\gamma-\sqrt{\frac{2 \varphi}{G\left(w^{*}\right)}}|f(X)|\right)} \cdot\left(\frac{\alpha_{P}-r}{\sigma_{P}^{2}}+\left(\gamma-\sqrt{\frac{2 \varphi}{G\left(w^{*}\right)}}|f(X)|\right) \frac{\partial g}{\partial X} \frac{\rho \xi}{\sigma_{P}}\right)
\end{aligned}
$$

where

$$
G(w)=\left(\sigma_{P}^{2} w^{2}+\xi^{2}\left(\frac{\partial g}{\partial X}\right)^{2}+2 w \rho \sigma_{P} \xi \frac{\partial g}{\partial X}\right) .
$$

Good market clearing implies

$$
P=\frac{e}{c} \quad, \quad \frac{d P}{P}=\frac{d e}{e}-\frac{d c}{c}-\frac{d c}{c} \frac{d e}{e}+\frac{d c}{c} \frac{d c}{c} .
$$

We have

$$
\begin{aligned}
d c & =\frac{\partial}{\partial X}\left(\frac{e^{\gamma g}}{\delta}\right)^{\frac{1}{\gamma-1}} d X+\frac{1}{2} \frac{\partial^{2}}{\partial^{2} X}\left(\frac{e^{\gamma g}}{\delta}\right)^{\frac{1}{\gamma-1}} d X^{2} \\
& =\frac{1}{\gamma-1}\left(\frac{e^{\gamma g}}{\delta}\right)^{\frac{1}{\gamma-1}} \gamma \frac{\partial g}{\partial X} d X+\frac{\gamma}{2(\gamma-1)}\left(\frac{\gamma}{\gamma-1}\left(\frac{e^{\gamma g}}{\delta}\right)^{\frac{1}{\gamma-1}}\left(\frac{\partial g}{\partial X}\right)^{2}+\left(\frac{e^{\gamma g}}{\delta}\right)^{\frac{1}{\gamma-1}} \frac{\partial^{2} g}{\partial^{2} X}\right) d X^{2} .
\end{aligned}
$$

and

$$
\begin{aligned}
\frac{d c}{c} & =\frac{\gamma}{\gamma-1} \frac{\partial g}{\partial X} d X+\frac{\gamma}{2(\gamma-1)}\left(\frac{\gamma}{\gamma-1}\left(\frac{\partial g}{\partial X}\right)^{2}+\frac{\partial^{2} g}{\partial^{2} X}\right) d X^{2} \\
& =\frac{\gamma}{\gamma-1}\left(\frac{\partial g}{\partial X} \zeta+\frac{1}{2}\left(\frac{\gamma}{\gamma-1}\left(\frac{\partial g}{\partial X}\right)^{2}+\frac{\partial^{2} g}{\partial^{2} X}\right) \xi^{2}\right) d t+\frac{\gamma}{\gamma-1} \frac{\partial g}{\partial X} \xi d Z^{X}
\end{aligned}
$$


This gives

$$
\frac{d c}{c} \frac{d e}{e}=\frac{\gamma}{\gamma-1} \frac{\partial g}{\partial X} \xi \rho_{e} \sigma_{e} d t \quad, \quad \frac{d c}{c} \frac{d c}{c}=\left(\frac{\gamma}{\gamma-1} \xi\right)^{2}\left(\frac{\partial g}{\partial X}\right)^{2}
$$

The cumulative return dynamics are then

$$
\begin{aligned}
\frac{d P+e d t}{P}= & {\left[\alpha_{e}+\frac{\gamma}{1-\gamma}\left(\frac{\partial g}{\partial X}\left(\zeta+\xi \rho_{e} \sigma_{e}\right)+\frac{1}{2}\left(\frac{\gamma}{1-\gamma}\left(\frac{\partial g}{\partial X}\right)^{2}+\frac{\partial^{2} g}{\partial^{2} X}\right) \xi^{2}\right)+\left(\frac{e^{\gamma g}}{\delta}\right)^{\frac{1}{\gamma-1}}\right] d t } \\
& +\left(\sigma_{e} \rho_{e}+\frac{\gamma}{1-\gamma} \frac{\partial g}{\partial X} \xi\right) d Z^{X}+\sigma_{e} \sqrt{1-\rho_{e}^{2}} d Z^{e}
\end{aligned}
$$

Solving for $\sigma_{P}^{2}$ the system of equations,

$$
\sigma_{P} \rho=\sigma_{e} \rho_{e}+\frac{\gamma}{1-\gamma} \frac{\partial g}{\partial X} \xi \quad, \quad \sigma_{P} \sqrt{1-\rho^{2}}=\sigma_{e} \sqrt{1-\rho_{e}^{2}},
$$

gives

$$
\sigma_{P}^{2}=\sigma_{e}^{2}+\frac{\gamma}{1-\gamma} 2 \rho_{e} \sigma_{e} \xi \frac{\partial g}{\partial X}+\left(\frac{\gamma}{1-\gamma} \xi\right)^{2}\left(\frac{\partial g}{\partial X}\right)^{2}
$$

Using the good and financial market clearing constraints the relevant single agent HJB equation becomes

$$
\begin{gathered}
0=\sup _{c, w}\left\{\frac{(c W)^{\gamma}-1}{\gamma}-\delta J+W\left(\alpha_{P}-c\right) J_{W}+\frac{1}{2} W^{2} \sigma_{P}^{2} J_{W W}+\zeta J_{X}+\frac{1}{2} \xi^{2} J_{X X}+W \rho \sigma_{P} \xi J_{X W}\right. \\
\left.-\sqrt{2 \varphi}|f(X)|\left[W^{2} \sigma_{P}^{2} J_{W}^{2}+\xi^{2} J_{X}^{2}+2 W \rho \sigma_{P} \xi J_{W} J_{X}\right]^{\frac{1}{2}}\right\}
\end{gathered}
$$

Adopting the shortened notation

it follows

$$
g^{\prime}=\frac{\partial g}{\partial X} \quad, \quad g^{\prime \prime}=\frac{\partial^{2} g}{\partial^{2} X}
$$

$$
\begin{aligned}
0= & \frac{\left(\frac{e^{\gamma g}}{\delta}\right)^{\frac{\gamma}{\gamma-1}} W^{\gamma}-1}{\gamma}-\frac{\left(e^{g} W\right)^{\gamma}-1}{\gamma}+\left(\alpha_{P}-\left(\frac{e^{\gamma g}}{\delta}\right)^{\frac{1}{\gamma-1}}\right) \frac{\left(e^{g} W\right)^{\gamma}}{\delta}+\frac{\gamma-1}{2} \sigma_{P}^{2} \frac{\left(e^{g} W\right)^{\gamma}}{\delta} \\
& +\zeta \frac{\left(e^{g} W\right)^{\gamma}}{\delta} g^{\prime}+\frac{1}{2} \xi^{2}\left(\gamma \frac{\left(e^{g} W\right)^{\gamma}}{\delta}\left(g^{\prime}\right)^{2}+\frac{\left(e^{g} W\right)^{\gamma}}{\delta} g^{\prime \prime}\right)+\gamma \rho \sigma_{P} \xi \frac{\left(e^{g} W\right)^{\gamma}}{\delta} g^{\prime} \\
& -\sqrt{2 \varphi}|f(X)|\left(\sigma_{P}^{2}\left(\frac{\left(e^{g} W\right)^{\gamma}}{\delta}\right)^{2}+\xi^{2}\left(\frac{\left(e^{g} W\right)^{\gamma}}{\delta}\right)^{2}\left(g^{\prime}\right)^{2}+2 \rho \sigma_{P} \xi\left(\frac{\left(e^{g} W\right)^{\gamma}}{\delta}\right)^{2} g^{\prime}\right)^{\frac{1}{2}} \\
= & \left(\frac{1}{\gamma}\left(\frac{e^{\gamma g}}{\delta}\right)^{\frac{1}{\gamma-1}}-\frac{\delta}{\gamma}+\alpha_{P}-\left(\frac{e^{\gamma g}}{\delta}\right)^{\frac{1}{\gamma-1}}+\frac{\gamma-1}{2} \sigma_{P}^{2}+\zeta g^{\prime}+\frac{1}{2} \xi^{2}\left(\gamma\left(g^{\prime}\right)^{2}+g^{\prime \prime}\right)+\gamma \rho \sigma_{P} \xi g^{\prime}\right) \frac{e^{\gamma g}}{\delta} \\
& -\sqrt{2 \varphi}|f(X)|\left(\sigma_{P}^{2}+\xi^{2}\left(g^{\prime}\right)^{2}+2 \rho \sigma_{P} \xi g^{\prime}\right)^{\frac{1}{2}} \frac{e^{\gamma g}}{\delta} \\
= & \frac{1}{\gamma}\left(\left(\frac{e^{\gamma g}}{\delta}\right)^{\frac{1}{\gamma-1}}-\delta\right)+\alpha_{P}-\left(\frac{e^{\gamma g}}{\delta}\right)^{\frac{1}{\gamma-1}}+\frac{\gamma-1}{2} \sigma_{P}^{2}+\left(\zeta+\gamma \rho \sigma_{P} \xi\right) g^{\prime}+\frac{1}{2} \xi^{2}\left(\gamma\left(g^{\prime}\right)^{2}+g^{\prime \prime}\right) \\
& -\sqrt{2 \varphi}|f(X)|\left(\sigma_{P}^{2}+\xi^{2}\left(g^{\prime}\right)^{2}+2 \rho \sigma_{P} \xi g^{\prime}\right)^{\frac{1}{2}} \cdot
\end{aligned}
$$


Inserting the equilibrium expressions

$$
\begin{aligned}
\alpha_{P} & =\alpha_{e}+\frac{\gamma}{1-\gamma}\left(g^{\prime}\left(\zeta+\xi \rho_{e} \sigma_{e}\right)+\frac{1}{2}\left(\frac{\gamma}{1-\gamma}\left(g^{\prime}\right)^{2}+g^{\prime \prime}\right) \xi^{2}\right)+\left(\frac{e^{\gamma g}}{\delta}\right)^{\frac{1}{\gamma-1}} \\
\sigma_{P}^{2} & =\sigma_{e}^{2}+\frac{\gamma}{1-\gamma} 2 \rho_{e} \sigma_{e} \xi g^{\prime}+\left(\frac{\gamma}{1-\gamma} \xi\right)^{2}\left(g^{\prime}\right)^{2} \\
\rho \sigma_{P} & =\rho_{e} \sigma_{e}+\frac{\gamma}{1-\gamma} \xi g^{\prime}
\end{aligned}
$$

we obtain

$$
\begin{aligned}
0= & \frac{1}{\gamma}\left(\left(\frac{e^{\gamma g}}{\delta}\right)^{\frac{1}{\gamma-1}}-\delta\right)+\alpha_{e}+\frac{\gamma}{1-\gamma}\left(g^{\prime}\left(\zeta+\xi \rho_{e} \sigma_{e}\right)+\frac{1}{2}\left(\frac{\gamma}{1-\gamma}\left(g^{\prime}\right)^{2}+g^{\prime \prime}\right) \xi^{2}\right) \\
& +\frac{\gamma-1}{2}\left(\sigma_{e}^{2}+\frac{\gamma}{1-\gamma} 2 \rho_{e} \sigma_{e} \xi g^{\prime}+\left(\frac{\gamma}{1-\gamma} \xi\right)^{2}\left(g^{\prime}\right)^{2}\right) \\
& +\left(\zeta+\gamma \xi\left(\rho_{e} \sigma_{e}+\frac{\gamma}{1-\gamma} \xi g^{\prime}\right)\right) g^{\prime}+\frac{1}{2} \xi^{2}\left(\gamma\left(g^{\prime}\right)^{2}+g^{\prime \prime}\right) \\
& -\sqrt{2 \varphi}|f(X)|\left(\sigma_{e}^{2}+\frac{\gamma}{1-\gamma} 2 \rho_{e} \sigma_{e} \xi g^{\prime}+\left(\frac{\gamma}{1-\gamma} \xi\right)^{2}\left(g^{\prime}\right)^{2}+\xi^{2}\left(g^{\prime}\right)^{2}+2\left(\rho_{e} \sigma_{e}+\frac{\gamma}{1-\gamma} \xi g^{\prime}\right) \xi g^{\prime}\right)^{\frac{1}{2}} \\
= & \frac{1}{\gamma}\left(\left(\frac{e^{\gamma g}}{\delta}\right)^{\frac{1}{\gamma-1}}-\delta\right)+\alpha_{e}+\frac{\gamma-1}{2} \sigma_{e}^{2}+\frac{1}{1-\gamma}\left(\zeta+\gamma \xi \rho_{e} \sigma_{e}\right) g^{\prime}+\frac{\gamma}{(1-\gamma)^{2}} \frac{\xi^{2}}{2}\left(g^{\prime}\right)^{2} \\
& +\frac{1}{1-\gamma} \frac{\xi^{2}}{2} g^{\prime \prime}-\frac{\sqrt{2 \varphi}}{(1-\gamma)}|f(X)|\left((1-\gamma)^{2} \sigma_{e}^{2}+2(1-\gamma) \rho_{e} \sigma_{e} \xi g^{\prime}+\left(\xi g^{\prime}\right)^{2}\right)^{\frac{1}{2}}
\end{aligned}
$$

Specifically, for the log utility case it follows

$$
0=\delta(\ln (\delta)-g)+\alpha_{e}-\frac{\sigma_{e}^{2}}{2}+\zeta g^{\prime}+\frac{\xi^{2}}{2} g^{\prime \prime}-\sqrt{2 \varphi}|f(X)|\left(\sigma_{e}^{2}+\xi^{2}\left(g^{\prime}\right)^{2}+2 \xi g^{\prime} \rho_{e} \sigma_{e}\right)^{\frac{1}{2}}
$$

This concludes the proof of the proposition.

Proof of Corollary 10. The financial markets clearing condition gives a reference model equity premium given by

$$
\widehat{\alpha}_{P}-\widehat{r}=\widehat{\sigma}_{P}^{2}-\left(\gamma-\sqrt{\frac{2 \varphi}{\widehat{G}(1)}}|f(X)|\right)\left(\widehat{\sigma}_{P}^{2}+\widehat{\rho} \xi \widehat{\sigma}_{P} \frac{\partial \widehat{g}}{\partial X}\right) .
$$

By subtracting the worst case drift contamination

$$
-\sqrt{\frac{2 \varphi}{\widehat{G}(1)}}|f(X)|\left(\widehat{\sigma}_{P}^{2}+\widehat{\rho} \xi \widehat{\sigma}_{P} \frac{\partial \widehat{g}}{\partial X}\right),
$$

we thus obtain a worst case model equity premium given by

$$
\left(\widehat{\alpha}_{P}-\widehat{r}\right)_{h^{*}}=\widehat{\sigma}_{P}^{2}-\gamma\left(\widehat{\sigma}_{P}^{2}+\widehat{\rho} \xi \widehat{\sigma}_{P} \frac{\partial \widehat{g}}{\partial X}\right)
$$


Proof of Proposition 12. We expand the differential equation (14), to fist order in $\gamma$ and $\sqrt{2 \varphi}$. This gives

$$
\begin{aligned}
0= & \frac{1}{\gamma}\left(\left(\frac{e^{\gamma g}}{\delta}\right)^{\frac{1}{\gamma-1}}-\delta\right)+\alpha_{e}+\frac{\gamma-1}{2} \sigma_{e}^{2}+\frac{1}{1-\gamma}\left(\zeta+\gamma \xi \rho_{e} \sigma_{e}\right) g^{\prime}+\frac{\gamma}{(1-\gamma)^{2}} \frac{\xi^{2}}{2}\left(g^{\prime}\right)^{2} \\
& +\frac{1}{1-\gamma} \frac{\xi^{2}}{2} g^{\prime \prime}-\frac{\sqrt{2 \varphi}}{(1-\gamma)}|f|\left((1-\gamma)^{2} \sigma_{e}^{2}+2(1-\gamma) \rho_{e} \sigma e \xi g^{\prime}+\left(\xi g^{\prime}\right)^{2}\right)^{\frac{1}{2}} \\
= & \delta \ln (\delta)-\delta g+\alpha_{e}-\frac{\sigma_{e}^{2}}{2}+\zeta g^{\prime}+\frac{\xi^{2}}{2} g^{\prime \prime}+\gamma \delta\left(\left(\frac{\widehat{g}_{\log , 0}}{2}-1-\ln (\delta)\right) \widehat{g}_{\log , 0}+\ln \delta\left(1+\frac{\ln \delta}{2}\right)\right) \\
& +\gamma\left(\frac{\sigma_{e}^{2}}{2}+\left(\zeta+\xi \rho_{e} \sigma_{e}\right) \widehat{g}_{\log , 0}^{\prime}+\frac{\xi^{2}}{2}\left(\widehat{g}_{\log , 0}^{\prime}\right)^{2}+\frac{\xi^{2}}{2} \widehat{g}_{\log , 0}^{\prime \prime}\right) \\
& -\sqrt{2 \varphi}|f|\left(\sigma_{e}^{2}+2 \rho_{e} \sigma_{e} \xi \widehat{g}_{\log , 0}^{\prime}+\left(\xi \widehat{g}_{\log , 0}^{\prime}\right)^{2}\right)^{\frac{1}{2}}+O^{2}(\gamma, \sqrt{\varphi}) .
\end{aligned}
$$

Expanding the candidate solution $\widehat{g}$ to first order,

$$
\widehat{g}=\widehat{g}_{\log , 0}+\gamma \widehat{g}_{1}+\sqrt{2 \varphi} \widehat{g}_{2}+O^{2}(\gamma, \sqrt{\varphi})
$$

and using the fact that $\widehat{g}_{\log , 0}$ is a solution of the zeroth order HJB equation it follows

$$
0=-\delta \widehat{g}_{1}+\zeta \widehat{g}_{1}^{\prime}+\frac{\xi^{2}}{2} \widehat{g}_{1}^{\prime \prime}+\mathbf{R}_{1}\left(\widehat{g}_{\log , 0}\right)
$$

where

$$
\mathbf{R}_{1}(g)=\delta\left(\left(\frac{g}{2}-1-\ln (\delta)\right) g+\ln (\delta)\left(1+\frac{\ln (\delta)}{2}\right)\right)+\frac{\sigma_{e}^{2}}{2}+\left(\zeta+\xi \rho_{e} \sigma_{e}\right) g^{\prime}+\frac{\xi^{2}}{2}\left(g^{\prime}\right)^{2}+\frac{\xi^{2}}{2} g^{\prime \prime}
$$

and

where

$$
0=-\delta \widehat{g}_{2}+\zeta \widehat{g}_{2}^{\prime}+\frac{\xi^{2}}{2} \widehat{g}_{2}^{\prime \prime}+R_{2}\left(\widehat{g}_{\log , 0}\right)
$$

$$
\mathbf{R}_{2}(g)=-\sqrt{2}|f|\left(\sigma_{e}^{2}+2 \rho_{e} \sigma_{e} \xi g^{\prime}+\left(\xi g^{\prime}\right)^{2}\right)^{\frac{1}{2}}
$$

This concludes the proof of the first statement. The second claim is obtained by first replacing $\widehat{g}_{\log , 0}$ with $\widehat{g}_{\log , \varphi}$ in the above expansions. This gives an inhomogenity $\mathbf{R}_{1}\left(\widehat{g}_{\log , \varphi}\right)$ and, when expanding $\mathbf{R}_{2}(\widehat{g})$ as

$$
\begin{aligned}
\mathbf{R}_{2}(\widehat{g})= & -\sqrt{2}|f|\left(\sigma_{e}^{2}+2 \rho_{e} \sigma_{e} \xi \widehat{g}^{\prime}+\left(\xi \widehat{g}^{\prime}\right)^{2}\right)^{\frac{1}{2}} \\
= & -\sqrt{2}|f|\left(\sigma_{e}^{2}+2 \rho_{e} \sigma_{e} \xi\left(\widehat{g}_{\log , \varphi}^{\prime}+\gamma \widehat{g}_{1}^{\prime}\right)+\xi^{2}\left(\widehat{g}_{\log , \varphi}^{\prime}+\gamma \widehat{g}_{1}^{\prime}\right)^{2}\right)^{\frac{1}{2}}+O\left(\gamma^{2}\right) \\
= & -\sqrt{2}|f|\left(\sigma_{e}^{2}+2 \rho_{e} \sigma_{e} \xi \widehat{g}_{\log , \varphi}^{\prime}+\left(\xi \widehat{g}_{\log , \varphi}^{\prime}\right)^{2}\right)^{\frac{1}{2}} \\
& -\gamma \sqrt{2}|f| \frac{\rho_{e} \sigma_{e}+\xi g_{\log , \varphi}^{\prime}}{\left(\sigma_{e}^{2}+2 \rho_{e} \sigma_{e} \xi \widehat{g}_{\log , \varphi}^{\prime}+\left(\xi \widehat{g}_{\log , \varphi}^{\prime}\right)^{2}\right)^{\frac{1}{2}}} \xi \widehat{g}_{1}^{\prime}+O\left(\gamma^{2}\right)
\end{aligned}
$$


the homogenous part of the differential equation for $\widehat{g}_{1}$ stated in the second claim of the proposition.

Proof of Corollary 13. We have to solve the differential equation (setting for brevity $a=a(\varphi)$ )

$$
\begin{aligned}
0= & -\delta g+\left(-\lambda(X-\bar{X})-\sqrt{2 \varphi} \xi \frac{\xi b+\rho_{e} \sigma_{e}}{\left(\sigma_{e}^{2}+2 \rho_{e} \sigma_{e} \xi b+(\xi b)^{2}\right)^{\frac{1}{2}}}\right) g^{\prime} \\
& +\frac{\xi^{2}}{2} g^{\prime \prime}+\delta\left(\left(\frac{a+b X}{2}-1-\ln (\delta)\right)(a+b X)+\ln (\delta)\left(1+\frac{\ln (\delta)}{2}\right)\right) \\
& +\frac{\sigma_{e}^{2}}{2}+\left(\lambda(\bar{X}-X)+\xi \rho_{e} \sigma_{e}\right) b+\frac{(\xi b)^{2}}{2} .
\end{aligned}
$$

When using a polynomial guess $g=\alpha+\beta X+\varepsilon X^{2}$ for a solution and when matching the resulting coefficients the solution is obtained.

Proof of Corollary 14. The proof is obtained with identical arguments as in the proof of Corollary 13.

Proof of Corollary 16. The proof is obtained with identical arguments as in the proof of Corollary 13.

Proof of (80). We have to compute

$$
f(X)=E_{X}\left(\frac{e_{0}}{e_{h}}\right):=E\left(\frac{e_{0}}{e_{h}} \mid X_{0}=X\right)
$$

based on the dynamics

$$
\begin{aligned}
d X & =\lambda(\bar{X}-X) d t+\xi d Z^{X} \\
\frac{d e}{e} & =X d t+\sigma_{e}\left[\rho_{e} d Z^{X}+\sqrt{1-\rho_{e}^{2}} d Z^{e}\right] .
\end{aligned}
$$

The normality of $\left(X_{t}\right)$ implies:

$$
\begin{aligned}
E_{X}\left(\frac{e_{0}}{e_{h}}\right) & =E_{X}\left(\exp \left(-\int_{0}^{h}\left(X_{t}+\frac{1}{2} \sigma_{e}^{2}\right) d t-\sigma_{e}\left[\rho_{e}\left(Z_{h}^{X}-Z_{0}^{X}\right)+\sqrt{1-\rho_{e}^{2}}\left(Z_{h}^{e}-Z_{h}^{e}\right)\right]\right)\right) \\
& =\exp \left(E_{X}\left(-\int_{0}^{h} X_{t} d t\right)+\frac{1}{2} \operatorname{Var}_{X}\left(\int_{0}^{h} X_{t} d t\right)\right) .
\end{aligned}
$$

Using the formulas

$$
\begin{aligned}
& E_{X}\left(-\int_{0}^{h} X_{t} d t\right)=\bar{X}\left(\left(\frac{1-\exp (-\lambda h)}{\lambda}\right)-h\right)-\left(\frac{1-\exp (-\lambda h)}{\lambda}\right) X \\
& \operatorname{Var}_{X}\left(\int_{0}^{h} X_{t} d t\right)=\frac{\xi^{2}}{\lambda^{2}} h-\frac{\xi^{2}}{\lambda^{3}}(1-\exp (-\lambda h))-\frac{\xi^{2}}{2 \lambda^{3}}(1-\exp (-\lambda h))^{2}
\end{aligned}
$$

concludes the proof. 


\section{References}

Abel, B. 1997 . An Exploration of the effects of Pessimism and Doubt on Asset Returns, Working Paper, Federal Reserve Bank of Philadelphia.

Anderson, E. W., L. P. Hansen, and T. J. Sargent, 2000 . Robustness, Detection and the Price of Risk, http://www.stanford.edu/ sargent/research.html.

Campbell, Y. C., and J. H. Cochrane, 1999 . By Force of Habit: a Consumption-Based Explanation of Aggregate Stock Market Behavior, Journal of Political Economy, vol 107, $2,205-251$.

Chan, L., C. and L. Kogan, 2001 . Catching up with the Joneses: Heterogeneous Preferences and the Dynamics of Asset Prices, Manuscript.

Chen, Z. and L. G. Epstein, 2000 . Ambiguity, Risk and Asset Returns in Continuous Time, mimeo, University of Rochester.

Dow, J., and R. Werlang 1992 . Uncertainty Aversion, Risk Aversion, and the Optimal Choice of Portfolio, Econometrica 60, 197-204.

Ellsberg, D., 1961 . Risk, Ambiguity and the Savage Axioms, Quarterly Journal of Economics, 25, 643-669.

Fama, E. F., and F., K. French, 1989 . Business Conditions and Expected Returns on Stocks and Bonds, Journal of Financial Economics, 25, 23-49..

Gilboa, I. and D. Schmeidler, 1989 . Maxmin Expected Utility with Non-unique Prior, Journal of Mathematical Economics, 18, 141-153.

Kim, T. S., and E. Omberg, 1996 . Dynamic Nonmyopic Portfolio Behavior, Review of Financial Studies, 9, 141-161.

Knight, F., 1921 . Risk, Uncertainty and Profit. Boston, Mass: Houghton Mifflin. Reprint, London: London School of Economics, 1946.

Kogan, L. and R. Uppal, 2000 . Risk Aversion and Optimal Policies in Partial and General Equilibrium Economies, http://www.finance.commerce.ubc.ca/ uppal/.

Johnsen, T., and J. B. Donanldson, 1985 . The Structure of Intertemporal Preferences under Uncertainty and Time Consistent Plans, Econometrica, vol. 53, no. 6, November 1985, pp. 1451-58.

Lei, C.I., 2001 , Why Don't Investors Have Large Positions in Stocks? A Robustness Perspective, Mimeo, Department of Economics, University of Chicago.

Maenhout, P., 1999 . Robust Portfolio Rules and Asset Pricing, http://www.economics.harvard.edu/ pmaenhou/papers.html.

Merton, R., 1969 . Lifetime Portfolio Selection Under Uncertainty: the Continuous Time Case, Review of Economics and Statistics, 51, 247-257.

Merton, R., 1971 . Optimum Consumption and Portfolio Rules in a Continuous-Time Model, Journal of Economic Theory, 3, 373-413. 
Trojani, F., and P. Vanini 2001a . Risk, Robustness and Knightian Uncertainty in Continuous Time, Heterogeneous Agents, Financial Equilibria, Working Paper, University of Southern Switzerland, Lugano, Switzerland.

Trojani, F., and P. Vanini 2001b . Perturbative Solutions of Hamilton Jacobi Bellman Equations in Non-Homothetic Robust Decision Making, Working Paper, University of Southern Switzerland, Lugano, Switzerland.

Trojani, F., and P. Vanini, 2002 . A Note on Robustness in Merton's Model of Intertemporal Consumption and Portfolio Choice, Journal of Economic Dynamics and Control, 26/3, p. $423-435$.

Uppal, R., and T. Wang (2000) . Model misspecification and under-diversification, Working paper, http://www.london.edu/faculty/ruppal.

Veronesi, P., 1999 . Stock Market Overreaction To Bad News In Good Times: A Rational Expectation Equilibrium Model, Review of Financial Studies, 12, 975-1007.

Veronesi, P., 2000 . How Does Information Quality Affect Stock Returns?", Journal of Finance, $55,807-837$.

Veldkamp, L., 2001 . Slow Boom, Sudden Crash, Manuscript, http://www.stanford.edu/ ${ }^{\sim}$ veldkamp/. 
Figure 1: Ornstein Uhlenbeck (OU) dynamics: Equity premia $\left(O\left(\gamma^{3}\right)\right.$-equilibrium asymptotics)
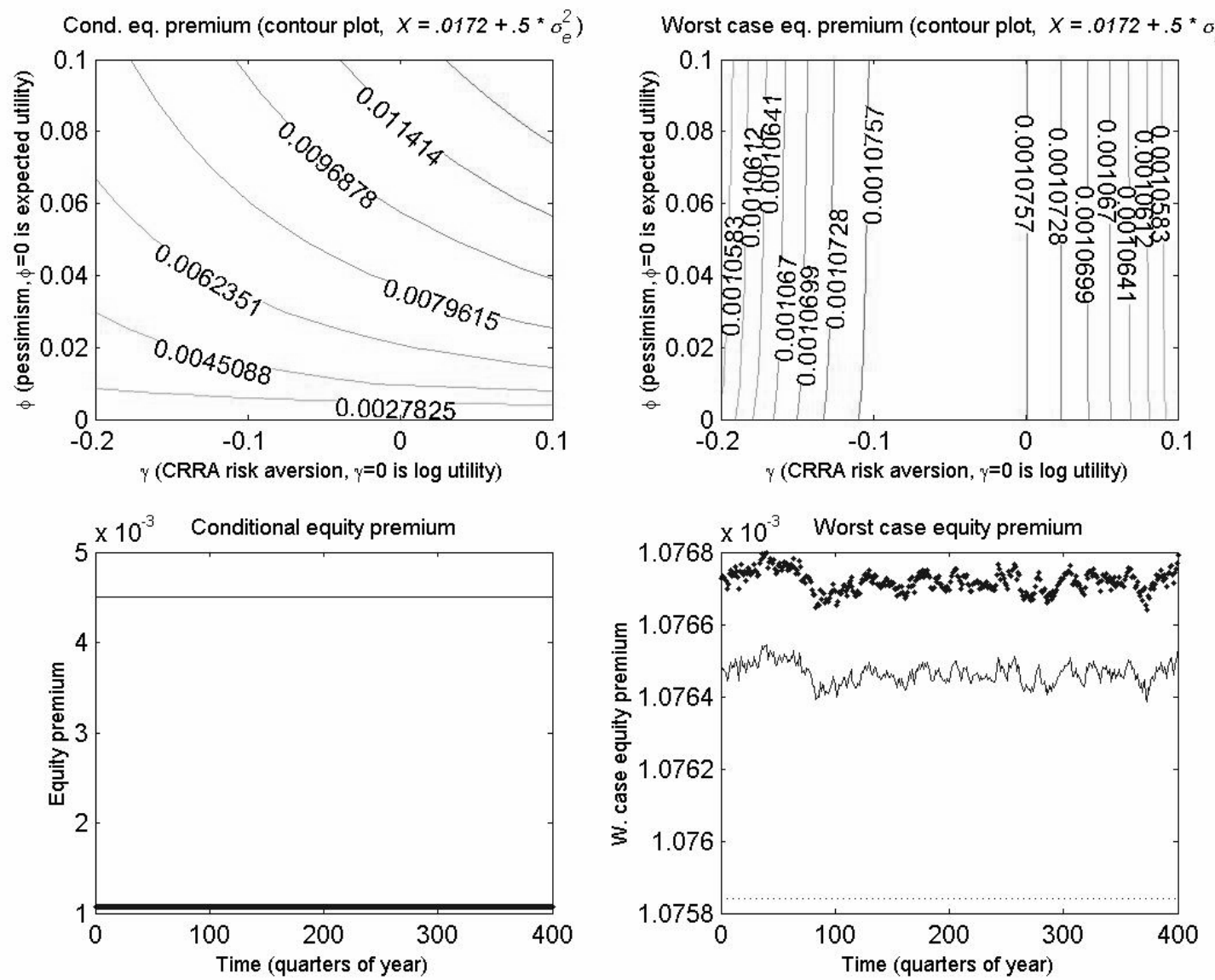

The equity premium, $\widehat{\alpha}_{P}-r$, and the worst case equity premium, $\left(\widehat{\alpha}_{P}-r\right)_{\widehat{h}^{*}}$, are shown according to the analytical $O\left(\gamma^{3}\right)$-equilibrium asymptotics. The economy has OU state dynamics for the expected dividend growth and constant pessimism, $f(X)=1$. Such dynamics is calibrated to US annual data on changes in the log real consumption. The calibrated dynamics is:

$$
\begin{aligned}
d X & =-0.5\left(X-\left(0.0172+0.5 \cdot 0.0382^{2}\right)\right) d t+0.0382 \cdot d Z^{X}, \\
d \ln e & =\left(X-0.5 \cdot 0.0382^{2}\right) d t+0.0382 \cdot\left[0.5 d Z^{X}+\sqrt{1-0.5^{2}} d Z^{e}\right],
\end{aligned}
$$

The time preference parameter $\delta$ is 0.053 . The dotted lines in the graphs correspond to the case $(\gamma=0, \varphi=0)$, the black-diamond lines to the case $(\gamma=-0.1, \varphi=0)$, and the straight lines to case $(\gamma=-0.1, \varphi=0.015)$. 
Figure 2: OU dynamics: Equity returns $\left(O\left(\gamma^{3}\right)\right.$-equilibrium asymptotics) and function $\widehat{g}$
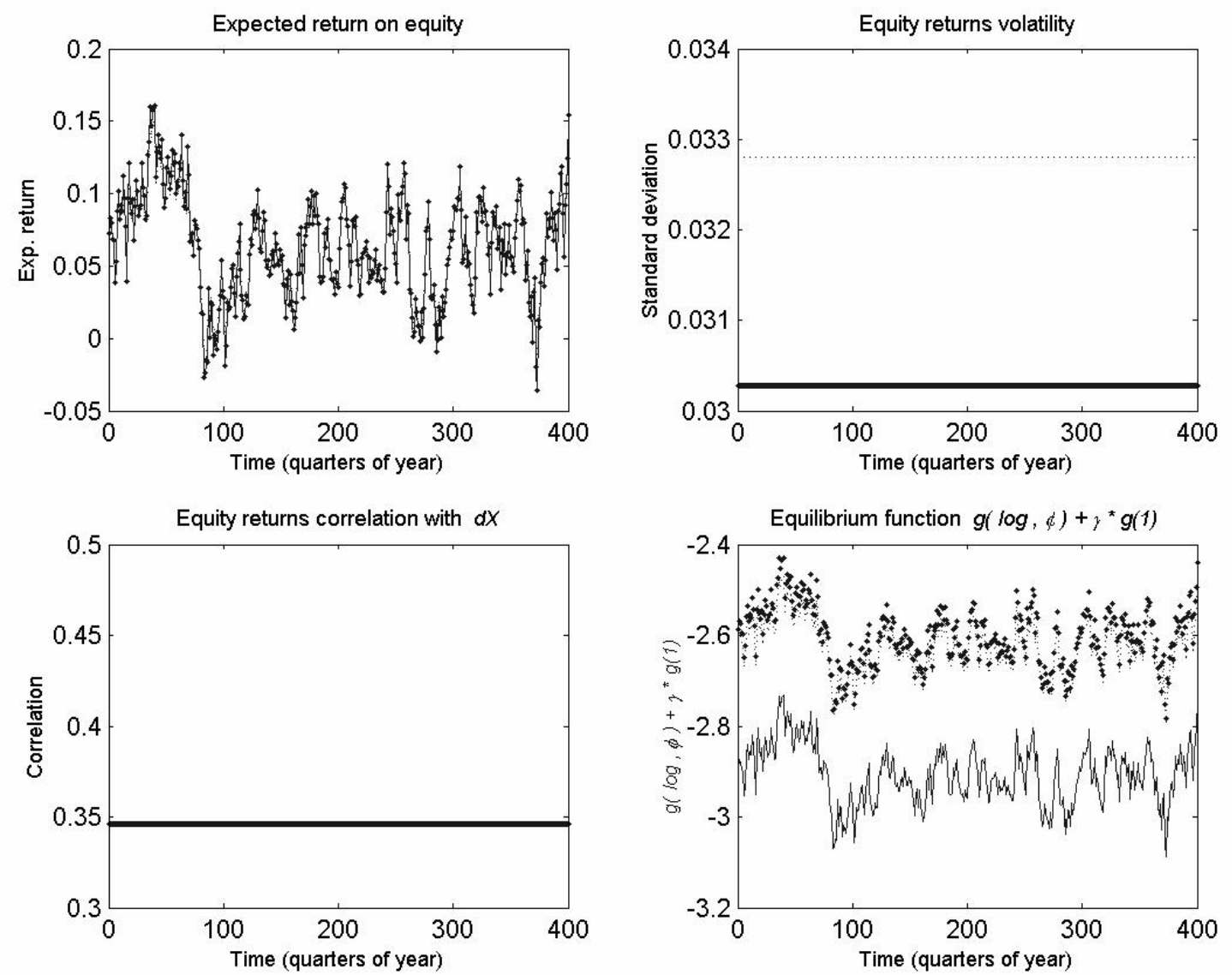

The time series of the conditional moments of equity returns, $\frac{d \widehat{P}+e d t}{\widehat{P}}$, and of the function $\widehat{g}$ are shown according to the analytical $O\left(\gamma^{3}\right)$-equilibrium asymptotics. $\widehat{g}$ is a key component of the equilibrium value function of a pessimistic representative agent, $\widehat{J}(\widehat{Y})=\frac{1}{\delta} \frac{\left(e^{\widehat{g}(X)} \widehat{P}\right)^{\gamma}-1}{\gamma}$. The time preference parameter $\delta$ is 0.053. The economy has OU state dynamics for the expected dividend growth and constant pessimism, $f(X)=1$. The calibrated model dynamics are the ones of Figure 1 . The time preference parameter $\delta$ is 0.053. The dotted lines in the graphs correspond to the case $(\gamma=0, \varphi=0)$, the black-diamond lines to the case $(\gamma=-0.1, \varphi=0)$, and the straight lines to the case $(\gamma=-0.1, \varphi=0.015)$. 
Figure 3: Cox Ingersoll Ross (CIR) dynamics: Equity premia $\left(O\left(\gamma^{3}\right)\right.$-equilibrium asymptotics)
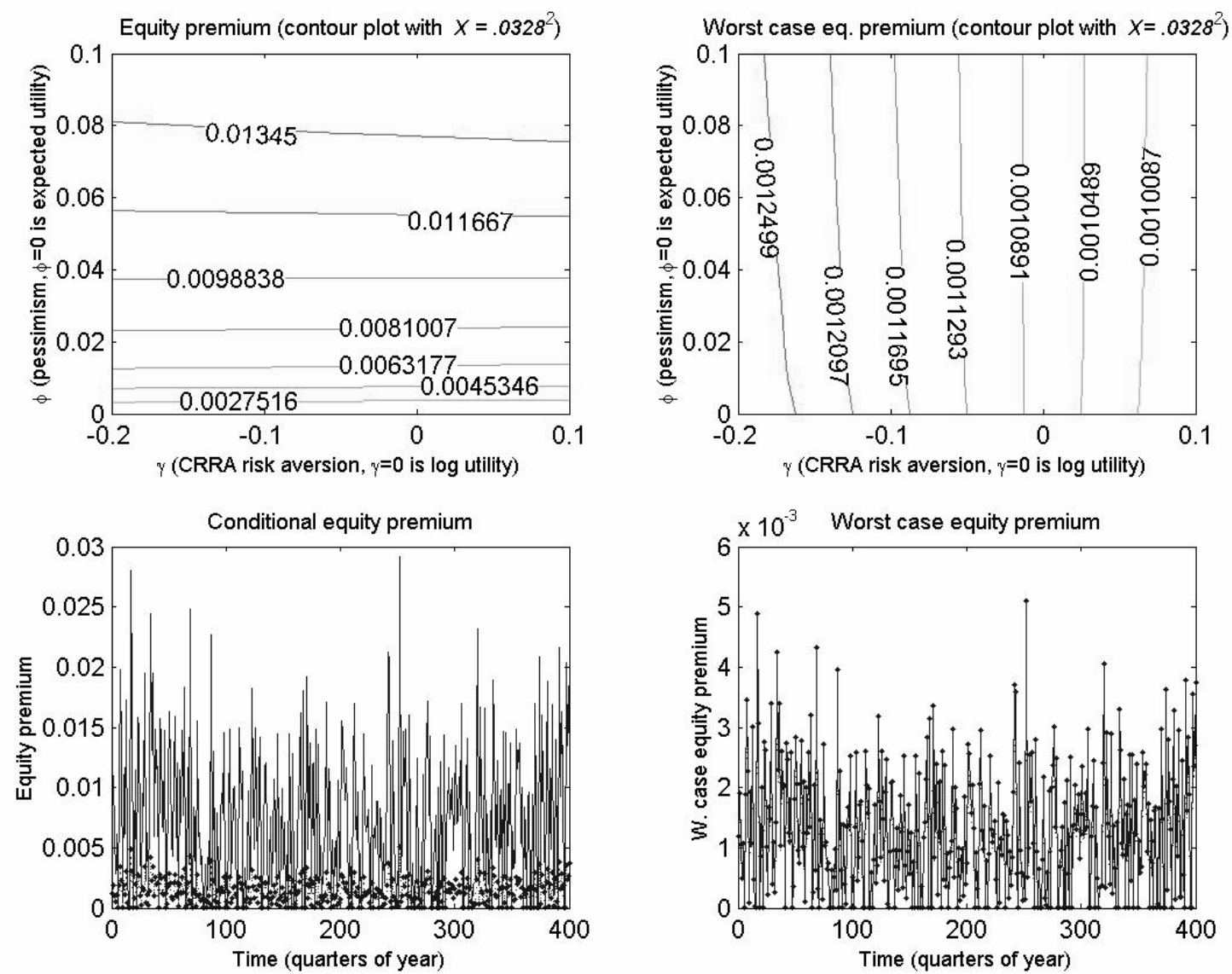

The equity premium, $\widehat{\alpha}_{P}-r$, and the worst case equity premium, $\left(\widehat{\alpha}_{P}-r\right)_{\widehat{h}^{*}}$, are shown according to the analytical $O\left(\gamma^{3}\right)$-equilibrium asymptotics. The economy has CIR state dynamics for the conditional variance of dividend growth and time varying pessimism, $f(X)=\sqrt{\frac{X}{\bar{X}}}$. Such dynamics is calibrated to US annual data on changes in the log real consumption. The calibrated dynamics is:

$$
\begin{aligned}
d X & =-3\left(X-(0.0382)^{2}\right) d t+0.0696 \cdot \sqrt{X} d Z^{X} \\
d \ln e & =\left(0.0177-0.5(1.00)^{2} X\right) d t+(1.00) \sqrt{X}\left[(-0.25) d Z^{X}+\sqrt{1-(-0.25)^{2}} d Z^{e}\right] .
\end{aligned}
$$

The time preference parameter $\delta$ is 0.053 . The dotted lines in the graphs correspond to the cases $(\gamma=0, \varphi=0)$, the black-diamond lines to the case $(\gamma=-0.1, \varphi=0)$, and the straight lines to the case $(\gamma=-0.1, \varphi=0.015)$. 
Figure 4: CIR dynamics: Equity returns $\left(O\left(\gamma^{3}\right)\right.$-equilibrium asymptotics) and function $\widehat{g}$
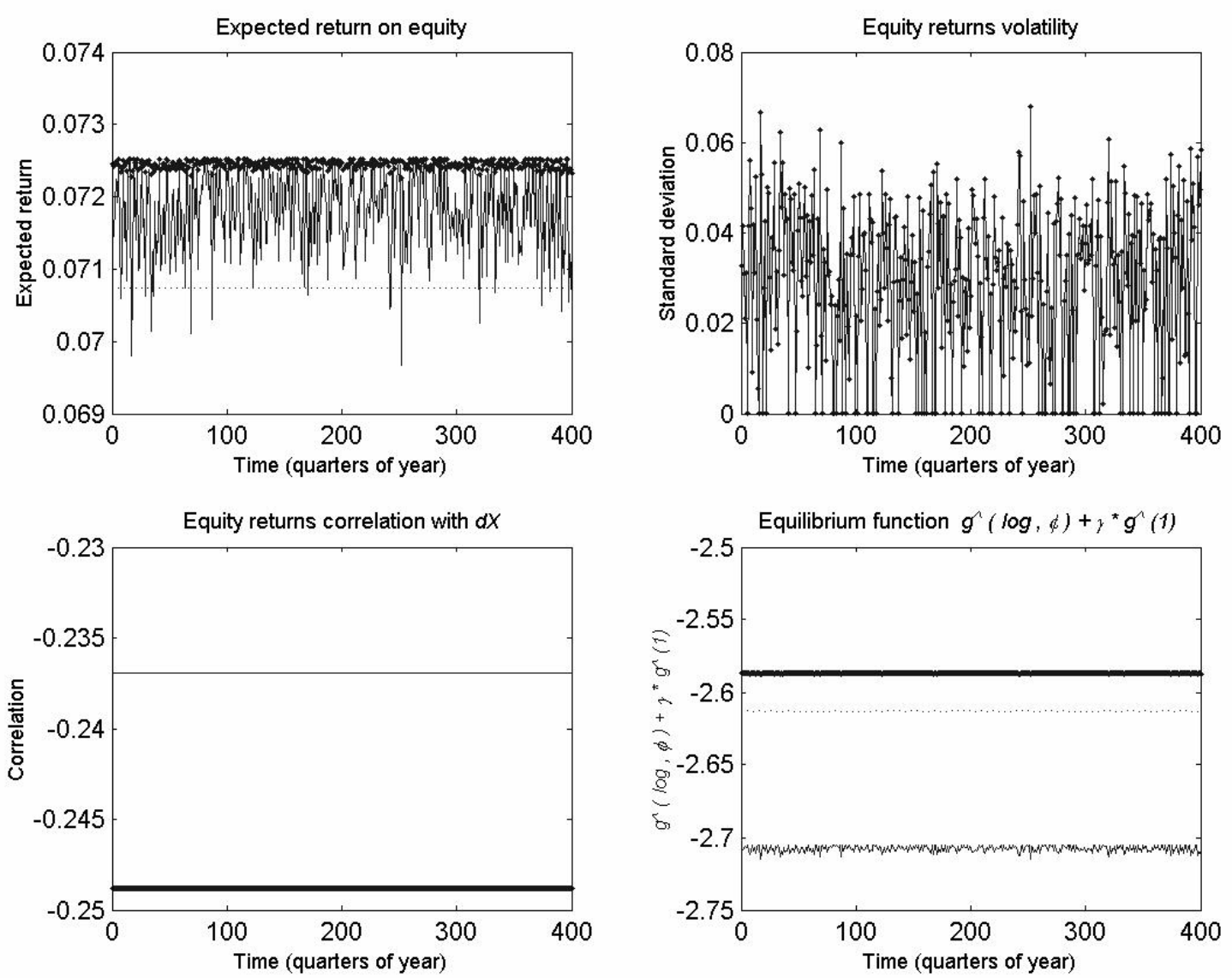

The time series of the conditional moments of equity returns, $\frac{d \widehat{P}+e d t}{\widehat{P}}$, and of the equilibrium function $\widehat{g}$ are shown according to the analytical $O\left(\gamma^{3}\right)$-equilibrium asymptotics. $\widehat{g}$ is a key component of the equilibrium value function of a pessimistic representative agent, $\widehat{J}(\widehat{Y})=\frac{1}{\delta} \frac{\left(e^{\widehat{g}(X)} \widehat{P}\right)^{\gamma}-1}{\gamma}$. The time preference parameter $\delta$ is 0.053 . The economy has CIR state dynamics for the conditional variance of dividend growth and time varying pessimism, $f(X)=\sqrt{\frac{X}{\bar{X}}}$. The calibrated dynamics are the ones of Figure 3 . The dotted lines in the graphs correspond to the case $(\gamma=0, \varphi=0)$, the black-diamond lines to the case $(\gamma=-0.1, \varphi=0)$, and the straight lines to the case $(\gamma=-0.1, \varphi=0.015)$. 
Figure 5: Geometric Ornstein Uhlenbeck (GOU) dynamics: Equity premia $\left(O\left(\gamma^{3}\right)\right.$-equilibrium asymptotics)
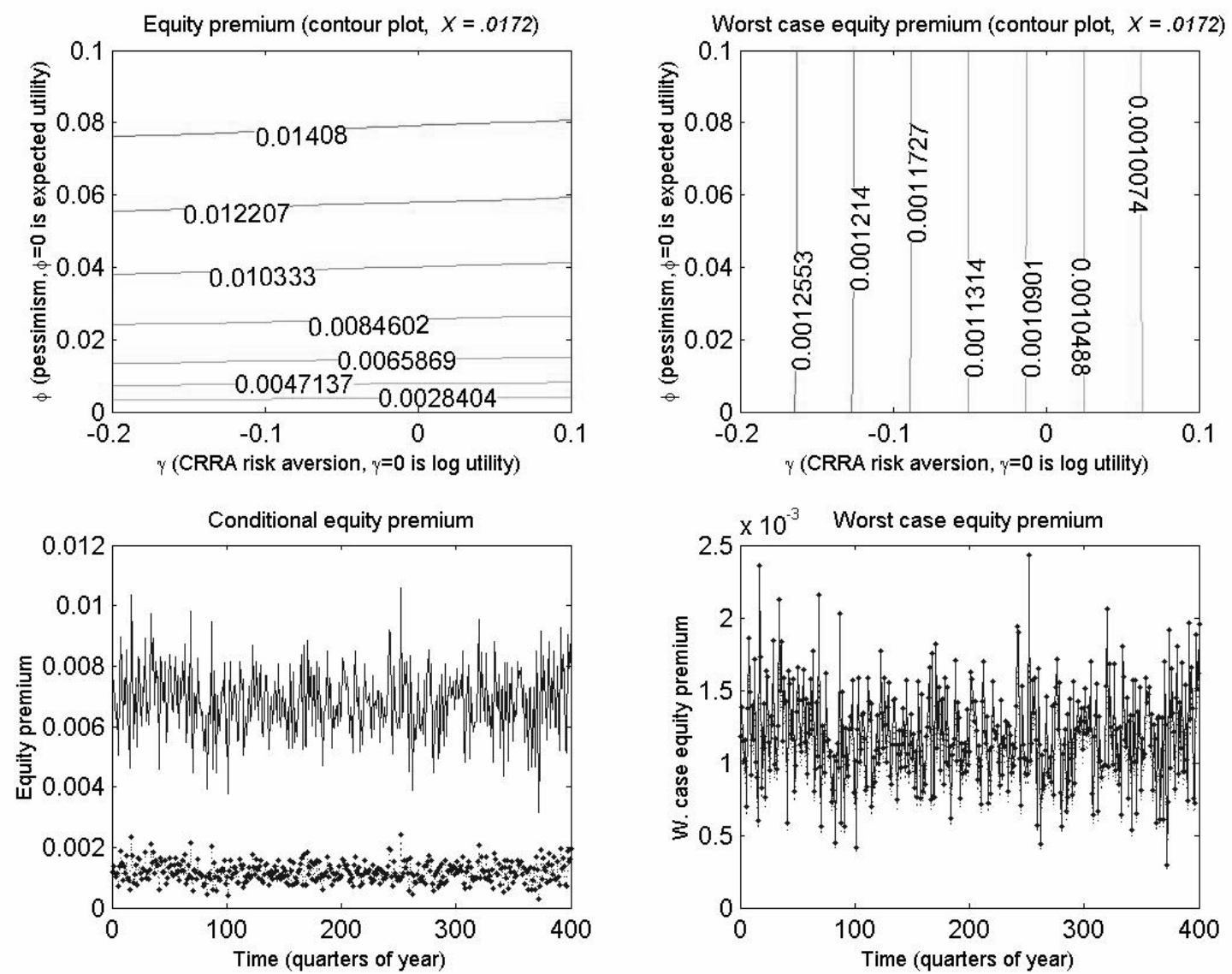

The equity premium, $\widehat{\alpha}_{P}-r$, and the worst case equity premium, $\left(\widehat{\alpha}_{P}-r\right)_{\widehat{h}^{*}}$, are shown according to the analytical $O\left(\gamma^{3}\right)$-equilibrium asymptotics. The economy has GOU state dynamics for the conditional mean and volatility of dividend growth, and it exhibits constant pessimism, $f(X)=1$. Such dynamics is calibrated to US annual data on changes in the log real consumption. The calibrated dynamics is::

$$
\begin{aligned}
d X & =-3(X-0.0172) d t+0.2782 \cdot X d Z^{X}, \\
d \ln e & =X d t+(0.0328 / 0.0172) X\left[0.1 d Z^{X}+\sqrt{1-0.1^{2}} d Z^{e}\right] .
\end{aligned}
$$

The time preference parameter $\delta$ is 0.053 . The dotted lines in the graphs correspond to the case $(\gamma=0, \varphi=0)$, the black-diamond lines to the case $(\gamma=-0.1, \varphi=0)$, and the straight lines to the case $(\gamma=-0.1, \varphi=0.015)$. 
Figure 6: GOU dynamics: Equity returns $\left(O\left(\gamma^{3}\right)\right.$-equilibrium asymptotics) and function $\widehat{g}$
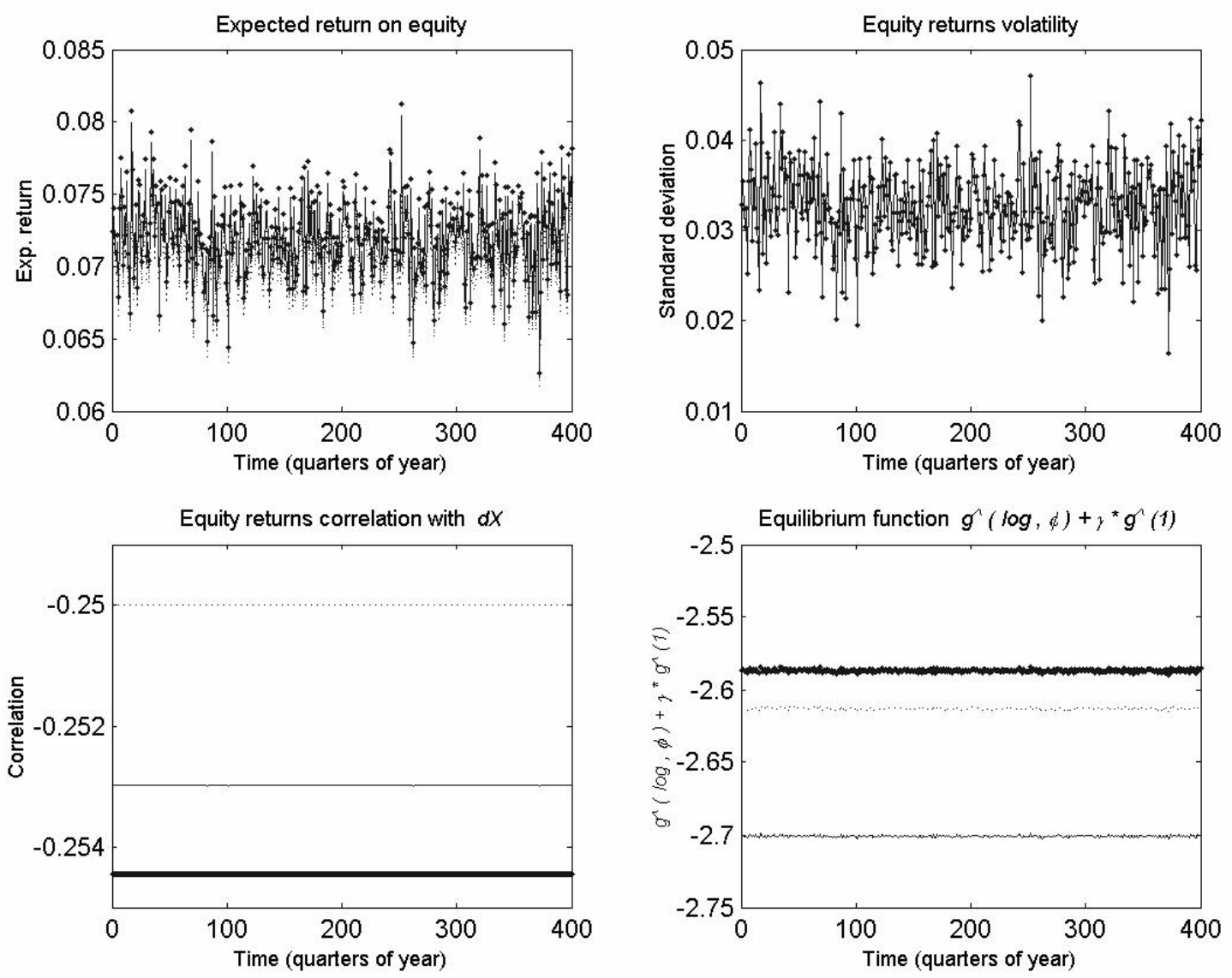

The time series of the conditional moments of equity returns, $\frac{d \widehat{P}+e d t}{\widehat{P}}$, and of the equilibrium function $\widehat{g}$ are shown according to the analytical $O\left(\gamma^{3}\right)$-equilibrium asymptotics. $\widehat{g}$ is a key component of the equilibrium value function of a pessimistic representative agent, $\widehat{J}(\widehat{Y})=\frac{1}{\delta} \frac{\left(e^{\widehat{g}(X)} \widehat{P}\right)^{\gamma}-1}{\gamma}$. The time preference parameter $\delta$ is 0.053 . The economy has GOU state dynamics for the conditional mean and volatility of dividend growth, and it exhibits constant pessimism, $f(X)=1$. The calibrated dynamics is the same of Figure 5 . The dotted lines in the graphs correspond to the case $(\gamma=0, \varphi=0)$, the black-diamond lines to the case $(\gamma=-0.1, \varphi=0)$, and the straight lines to the case $(\gamma=-0.1, \varphi=0.015)$. 
Figure 7: OU dynamics: Equity premia $\left(O^{2}(\gamma, \sqrt{\varphi})\right.$-equilibrium asymptotics)
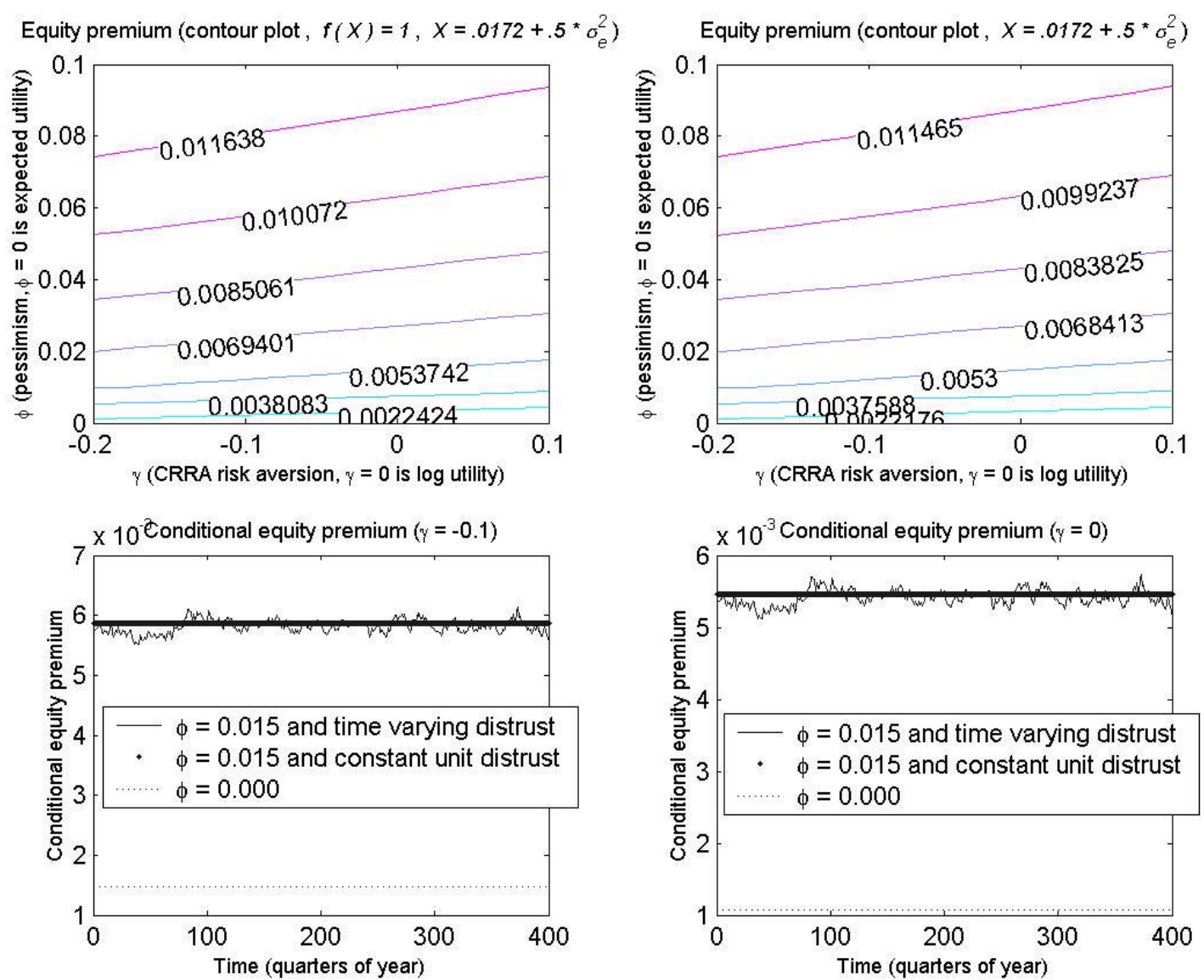

The contour plot and the time series of the equity premium, $\widehat{\alpha}_{P}-r$, are shown according to the analytical $O^{2}(\gamma, \sqrt{\varphi})$-equilibrium asymptotics. The economy has OU state dynamics for the expected dividend growth and time varying pessimism, $f(X)=E\left(\frac{e_{0}}{e_{h}} \mid X_{0}=X\right)$ with $h=1$. The calibrated dynamics is the same of Figure 1. The time preference parameter $\delta$ is 0.053 . 
Figure 8: CIR dynamics: Equity premia $\left(O^{2}(\gamma, \sqrt{\varphi})\right.$-equilibrium asymptotics)
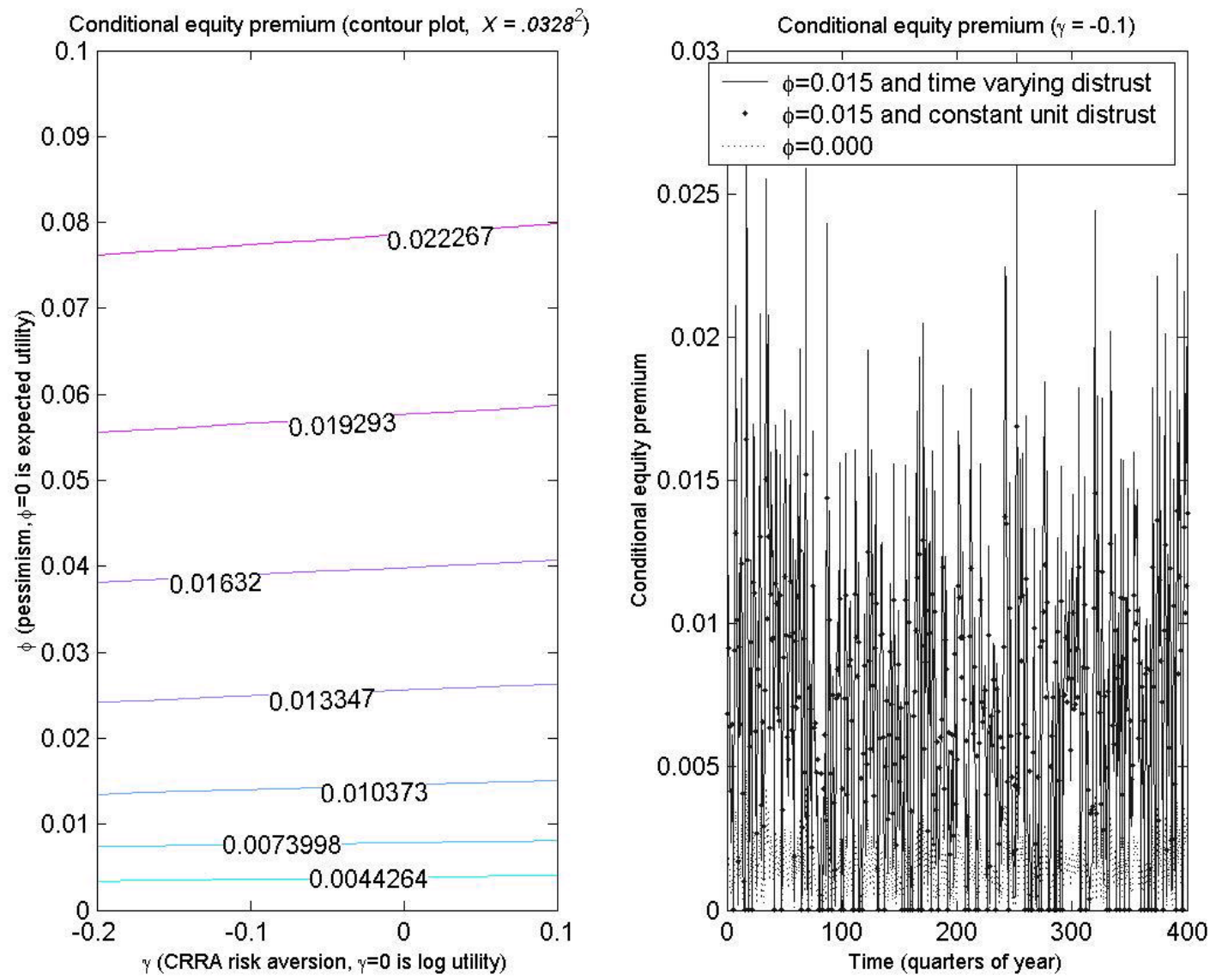

The contour plot and the time series of the equity premium, $\widehat{\alpha}_{P}-r$, are shown according to the analytical $O^{2}(\gamma, \sqrt{\varphi})$-equilibrium asymptotics. The economy has OU state dynamics for the expected dividend growth and time varying pessimism, $f(X)=\left(\frac{h \cdot \operatorname{Var}_{0}\left(\frac{d e}{e}\right)}{E_{0}\left(\int_{0}^{h} \operatorname{Var}_{t}\left(\frac{d e}{e}\right) d t \mid X_{0}=X\right)}\right)$ with $h=1$. The calibrated dynamics is the same of Figure 3 .The time preference parameter $\delta$ is 0.053 . 